\title{
Solidification of binary aqueous solutions under periodic cooling. Part 2. Distribution of solid fraction
}

\author{
Guang-Yu Ding ${ }^{1,3}$, Andrew J. Wells ${ }^{2}$ and Jin-Qiang Zhong ${ }^{1} \dagger$ \\ ${ }^{1}$ School of Physics Science and Engineering, Tongji University, Shanghai, China \\ ${ }^{2}$ Department of Physics, University of Oxford, Oxford, UK \\ ${ }^{3}$ Department of Physics, Chinese University of Hong Kong, Hong Kong, China
}

(Received xx; revised xx; accepted xx)

We report an experimental study of the distributions of temperature and solid fraction of growing $\mathrm{NH}_{4} \mathrm{Cl}-\mathrm{H}_{2} \mathrm{O}$ mushy layers that are subjected to periodical cooling from below, focussing on late-time dynamics where the mushy layer oscillates about an approximate steady state. Temporal evolution of the local temperature $T(z, t)$ at various heights in the mush demonstrates that the temperature oscillations of the bottom cooling boundary propagate through the mushy layer with phase delays and substantial decay in the amplitude. As the initial concentration $C_{0}$ increases, we show that the decay rate of the thermal oscillation with height also decreases, and the propagation speed of the oscillation phase increases. We interpret this as a result of the solid fraction increasing with $C_{0}$, which enhances the thermal conductivity but reduces the specific heat of the mushy layer. We present a new methodology to determine the distribution of solid fraction $\phi(z)$ in mushy layers for various $C_{0}$, using only measurements of the temperature $T(z, t)$. The method is based on the phase behaviour during thermal modulation, and opens up a new approach to inferring mushy layer properties in geophysical and engineering settings, where direct measurements are challenging. In our experiments, profiles of the solid fraction $\phi(z)$ exhibit a cliff-ramp-cliff structure with large vertical gradients of $\phi$ near the mush-liquid interface and also near the bottom boundary, but much more gradual variation in the interior of the mushy layer. Such a profile structure is more pronounced for higher initial concentration $C_{0}$. For very low concentration, the solid fraction appears to be linearly dependent on the height within the mush. The volume-average of the solid faction, and the local fluctuations in $\phi(z)$ both increase as $C_{0}$ increases. We suggest that the fast increase of $\phi(z)$ near the bottom boundary is possibly due to diffusive transport of solute away from the bottom boundary and the depletion of solute content near the basal region.

Key words: solidification/melting, convection in porous media, sea ice

\section{Introduction}

Understanding the fundamental physics of a solidifying aqueous binary fluid is important in a wide range of sciences, including geophysics, oceanography, metallurgy and electrical engineering (Loper \& Roberts 1983; Hobbs 2010; Chalmers 1964; Rosenberger

$\dagger$ Email address for correspondence: jinqiang@tongji.edu.cn 
1979). When a binary fluid is cooled and solidified from a horizontal plane boundary, the growing solid phase can form a dendritic structure of crystals called a mushy layer (see part I, Ding et al. 2018, for a more detailed discussion). Mushy layers are reactive porous materials with interstitial melt that can flow in response to externally applied forces. The dynamics of the interstitial flow are resisted by the porous structure, which depends on the local solid fraction $\phi$, the volume ratio of the solid to the mixture. The porosity $1-\phi$ is simply related to the solid fraction.

Since the liquid and solid phases have different thermal and mechanical properties (such as heat conductivity, heat capacity etc), the field equations that describe the evolution of temperature and melt concentration within the mushy layers involve governing parameters strongly dependent on $\phi$. Accurate information about $\phi$, including its variation in both space and time, thus plays a crucial role to improve theoretical modelling of mushy-layer growth. Early theoretical work by Huppert \& Worster (1985) considered the solid fraction as an important parameter in the governing equations for mushy-layer growth. This pioneering theoretical model treated the mushy layer as a continuum, with the physical properties of the solid and liquid being averaged over the mush region. It predicted the growth rate, temperature and concentration profiles of mushy layers that were in agreement with experimental results. In more advanced theoretical models (e.g. Worster 1986, 1991) predictions were made for the solid fraction evolving in space and time. The large specific surface area allows interstitial gradients of heat and solute to relax rapidly to a state of local thermodynamic equilibrium, with the temperature and concentration in the liquid pore spare coupled via a liquidus constraint. Then, knowledge of the bulk phase-weighted concentration and temperature is sufficient to predict the solid fraction $\phi$ (see Worster 2000, for a summary). Thus macroscopic temperature changes or solute transport through the mushy layer can modify the solid fraction. A particularly striking modification of solid fraction arises from convective flow within the mushy layer, which can dissolve solid-free channels or chimneys in the mushy layer, through which buoyant plumes of fluid drain into the neighbouring fluid region (see reviews by Worster 1997; Zhong et al. 2012). This process is sometimes called gravity drainage, and is a primary mechanism for solute fluxes from a growing mushy layer (Notz \& Worster 2009) providing a coupled feedback on mushy layer solid fraction and permeability. Experimental studies of the solid fraction with adequate resolution in both space and time will advance our understanding of the fluid dynamics involved in solidifying binary fluids.

Due to the fact that mushy layers are porous materials that consist of solid crystals, they are optically opaque. Direct measurements of the solid fraction in mushy layers through optical methods are challenging. Moreover, since growing mushy layers have a three-dimensional dendritic structure on multiple scales (Huppert 1990; Golden et al. 2007 ) determining $\phi$ through statistical analysis of experimental data requires high resolution measurement, and it is particularly challenging to capture porosity evolution during growth. In early experimental studies, Chen \& Chen (1991) and Chen (1995) applied X-ray tomography to measure the solid fraction in a mushy layer using a postmortem measurement after solidifying an aqueous ammonium chloride solution. It was observed that the solid fraction decreases significantly towards the mush-liquid interface. Wettlaufer et al. (1997) determined the time-evolution of the vertically-averaged solid fraction in an aqueuous sodium chloride mushy layer by measuring the volumetric change during solidification due to the density difference between water and ice. A variety of experimental systems have been used to show that the melting of a reactive mixed phase region can result in solidification deeper within that region as a result of convection, and investigated the evolution of the solid fraction (Hallworth \& Huppert 2004; Hallworth 
et al. 2005; Yu et al. 2015; Huguet et al. 2016). Several authors (Shirtcliffe et al. 1991; Chiareli \& Worster 1992; Notz et al. 2005) developed methods to measure the electrical resistance in the melt, which varies with the local solid fraction and allows the evolution of $\phi(z)$ to be determined as it evolves during solidification. This method has been extended and employed for in-situ field measurements of sea ice (Notz \& Worster 2008, 2009) which is a geophysical example of a mushy layer. Such a method was also successfully applied to the growth of a mushy layer under high gravity (Huguet et al. 2016). Bulk concentration and solid fraction have also been inferred from measurements of the complex dielectric permittivity and temperature of sea ice using embedded probes (Backstrom \& Eicken 2006), via surface impedance tomography (Sampson et al. 2011), or via electromagnetic induction (Hunkeler et al. 2015). Aussillous et al. (2006) used magnetic resonance imaging (MRI) to study how the fine structure and solid fraction evolves during solidification of a mushy layer. The solid fraction increases in the lower part of the layer after the onset of internal convection. Finally, we note that a range of post-mortem measurements have been taken from cores of sea ice, which is necessarily destructive, as the sample is removed for analysis. Solid fraction can be inferred from measured ice temperature, assumed local thermodynamic equilibrium, and bulk salinity measured from melted slices of the sample (e.g. see discussion in Hunke et al. 2011), or via scanning methods using MRI or micro-computed tomography (e.g. Eicken et al. 2000; Lieb-Lappen et al. 2017).

Despite a variety of methods have been attempted, very few high-resolution measurements have been made to determine the distribution of solid fraction and its evolution in growing mushy layers through well-controlled laboratory experiments. To date, all such measurements used either destructive post-mortem sampling, freezing of instruments into a growing mushy layer, or encounter difficulties of growing small samples of mushy layer within the confines of an imaging scanner. In this paper, we report experimental studies of the profiles of solid fraction $\phi$ in a mushy layer formed when an aqueous ammonium chloride solution is cooled from below with a periodically varying boundary temperature. We measure the local temperature $T(z, t)$ at various heights within the mushy layer, and characterise how the phase of temperature oscillations propagate from the bottom boundary through the mushy layer, and how the amplitude decays with height. We show that the decay rate of the thermal oscillation decreases with an increasing initial concentration $C_{0}$, but the propagation speed of the oscillation phases increases with $C_{0}$. These behaviours are consistent with an increase in solid fraction which enhances the thermal conductivity but reduces the specific heat of the mushy layer. Using high-resolution measurements of $T(z, t)$ we present a method for determining the distribution of solid fraction $\phi(z)$ for various $C_{0}$, based on the dependence of thermal material properties on $\phi$. Our analysis of the time-dependent heat transport equation reveals that when the mush temperature varies periodically in response to the modulation of the cooling boundary, the increment of the fluid internal energy can be appropriately correlated to the local conductive heat transports, as presented in a heatflux diagram. With these experimental findings, we extract accurate results of the local thermal diffusivity and hence determine the distribution of the solid fraction $\phi(z)$ in the mushy layer. Our approach provides a method to estimate solid fraction using only measurements of temperature within the mushy layer.

In the next section, we summarise the apparatus, experimental methods, and procedures that are described in more detail in Part I (Ding et al. 2018), which considered the early time growth characteristics of a mushy layer with the boundary temperature modulated periodically in time. In section 3 we describe experimental measurements of temperature during the corresponding late-time period of mushy layer growth, where the mushy layer is modulated about a nearly steady background state. 
The results compare well to a simplified theoretical model for thermal wave propagation due to diffusive heat transfer with uniform material properties (derived in appendix B). In $\S 4$ we analyse heat transfer within the mushy layer, outlining an algorithm to estimate the solid fraction via the thermal properties, and investigate how the solid fraction varies in space and time. The mean solid fraction estimated from mushy layer temperature variation compares well to an independent estimate from global solute conservation and measurements of concentration in the overlying liquid. In $\$ 5$ we summarise the results and discuss the implications for future work.

\section{Experimental methods}

The construction of the apparatus and the experimental environment have been described in detail in Part 1 (Ding et al. 2018). Here we briefly review the experimental procedure, and introduce the experimental components and methods that are relevant to our temperature and concentration measurements. A thermally shielded $80 \mathrm{~mm} \times 80 \mathrm{~mm}$ $\times 150 \mathrm{~mm}$ tank of aqueous $\mathrm{NH}_{4} \mathrm{Cl}$ solution is cooled from below, with the bottom temperature $T_{b}(t)=T_{b, 0}+A \sin \left(\omega t+\phi_{0}\right)$ controlled and modulated over time $t$ about a background temperature $T_{b, 0}$, with amplitude $A$, angular frequency $\omega$ and initial phase $\phi_{0}$. A vertically stacked array of thermistors measures temperature $T(z, t)$ at different heights $z$ in the mushy layer, and shadowgraphy images were analysed to infer the mush thickness $h(t)$. See Part 1 (Ding et al. 2018) for further details on the configuration, methods used, and procedure for initializing the experiment.

Fluid concentration and temperature within the liquid region were measured by a dual probe consisting of a four-electrode microscale conductivity sensor and a thermocouple (MSCTI, Precision Measurement Engineering, model 125). The probe was mounted on a computer-controlled positioner. Its conductivity sensor and the thermocouple junction were located at the same vertical level and $3 \mathrm{~mm}$ apart in the horizontal. When measuring the vertical profiles of $C(z)$ and $T(z)$, the dual probe traversed vertically through the liquid region at a constant speed of $0.4 \mathrm{~mm} / \mathrm{s}$ (Fig. 1a). Both the conductivity and temperature signals were recorded at a sampling frequency of $1 \mathrm{~Hz}$, yielding a vertical resolution of $0.4 \mathrm{~mm}$ for each profile. In each profile measurement the probe travelled $80 \mathrm{~mm}$, taking 200 seconds. Such a time span is at least one order in magnitude less than the modulation period $\tau=2 \pi / \omega$, and is much less than the time scale of mushylayer growth. During the measurements data were always recorded while the probe was traversed downwards, with the two sensors ahead of the probe holder, to minimize any disturbances in the measured region. Several scans were made within each modulation period to determine the mean solid fractions $\langle\phi\rangle$ as we discuss in $§ 4.4$.

The conductivity sensor and the thermocouple were calibrated before and after each experiment against $\mathrm{NH}_{4} \mathrm{Cl}$ solutions of known concentrations at various temperatures that cover the entire experimental parameter range, with an accuracy of $0.1 w t \%$ in concentration and $0.02 \mathrm{~K}$ in temperature, respectively. The voltage signal of the thermocouple was first turned into temperature using a fitted six-order polynomial function. The output voltage from the conductivity sensor was then translated into concentration based on the calibrated conductivity-concentration relations (Head 1983). Measurements of $C(z)$ and $T(z)$ were made in late modulation periods with $t \geqslant 5 \tau$ with mush temperature measurements performed at the same time. In these late periods, our temperature measurements indicate that the thermal properties and hence the porosity structure of the mushy layers show no long-term trend, with the signal repeatable between modulation periods (Fig. 2 and Fig. 10). As discussed in $\S 4.4$, we estimate the mean solid fraction $\langle\phi\rangle$ 

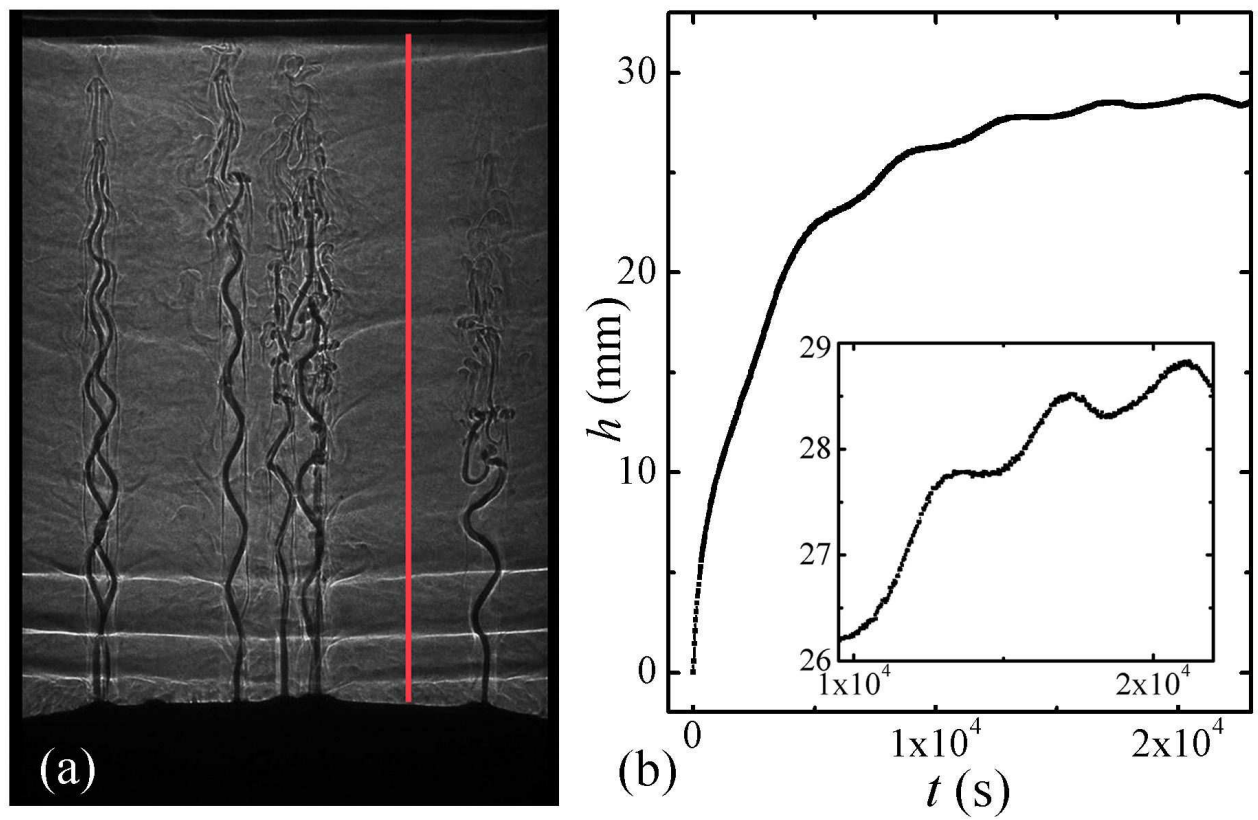

Figure 1. (a) Shadowgraph image that shows the mush region (black area near the bottom), narrow salt-finger plumes near the mush-liquid interface, wider plumes rising from chimneys and the double-diffusive layers in the liquid region. The image is captured at the beginning of the 6th modulation period in an experiment with initial concentration $C_{0}=29 w t \%, A=10 \mathrm{~K}$ and $\tau=4000 \mathrm{~s}$. The vertical red line marks the path along which the MSTCI probe traverses when temperature and salinity measurements are taken in the liquid region. (b) The mushy-layer height $h$ as a function of time. Inset: a segment of $h(\mathrm{t})$ from the 4 th to the 6th modulation periods.

in the mushy region, applying conservation of solute and using the concentration profile measured in the liquid region. These results for $\langle\phi\rangle$ are compared with an alternative estimated based on our temperature measurements within the mushy layers.

Various initial liquid concentrations $C_{0}$ were initially investigated. For a better resolution in measuring the mushy-layer thickness $h(t)$ and the mush temperature $T(z, t)$, we chose $C_{0}$ in the range of $26 w t \% \leqslant C_{0} \leqslant 32 w t \%$. The solution was prepared at an initial temperature $T_{0}$ above the liquidus temperature $T_{0}>T_{L}\left(C_{0}\right)$ to avoid crystallization prior to the experiment.

\section{The mushy-layer growth and the temperature distribution}

The dynamical growth of mushy layers in early periods of periodic cooling has been discussed in Part I (Ding et al. 2018) revealing modulation of the mushy layer thickness around a background state in the absence of modulation. The experimental data of the mushy-layer thickness $h(t)$ were shown to be broadly consistent with theoretical predictions from a numerical model of mushy-layer growth. In this section we present measurements of $h(t)$ and the temperature distribution $T(z, t)$ within the mushy layers at relatively late modulation periods when the mushy layer nearly approaches a constant thickness. We also discuss how temperature oscillations propagate through the mushy layer in this state.

The shadowgraph image in Fig. 1a presents the typical morphology of the mushylayer and flow structures in the liquid region during the late modulation periods. As is 
commonly seen when $\mathrm{NH}_{4} \mathrm{Cl}-\mathrm{H}_{2} \mathrm{O}$ solutions are cooled from below (e.g. see review by Zhong et al. 2012) the shadowgraph reveals multiple buoyant solute depleted plumes rising from chimneys in the mushy layer, with smaller solute-depleted plumes near the mush-liquid interface. In section 4.4 we suggest that the white near horizontal bands in the background fluid are likely the result of double-diffusive layering due to the cold plumes cooling a solute stratification. Formation of double-diffusive layers has also been observed in earlier experiments of solidification of a mushy layer (Beckermann \& Viskanta 1988; Chen 1997). The speed at which the mush-liquid interface advances towards the liquid region is obtained through our image processing method using a sequence of shadowgraph images (see discussions in Part. I Ding et al. 2018). Fig. 1b depicts results of the mushylayer height $h(t)$ from an experiment with an initial concentration $C_{0}=29 w t \%$. It is clearly seen that oscillations appear in $h(t)$ about a background growth curve which at early times is reminiscent of the scaling $h_{0}(t) \propto t^{1 / 2}$ characteristic of diffusive growth into a deep fluid layer with constant basal cooling. In the later periods the oscillation amplitude decays and the mean of $h(t)$ finally becomes nearly independent on time (inset of Fig. 1b) with small oscillations about a nearly steady state. As we will elucidate in detail in the following sections, during the late growth periods convective heat transport through the mushy layer becomes negligible. Therefore the growth of the mushy layer is dictated mainly by thermal conduction and its advance speed is determined by the thermal gradient at the mush-liquid interface. The mush thickness eventually reaches a statistically steady state where the heat flux from the mush balances the turbulent heat flux from compositional convection at the mush-liquid interface. Modulated by the cooling boundary, the mush temperature and thus the thermal gradient varies periodically in time with a nearly constant mean, and gives rise to the periodic oscillations of the growth curve $h(t)$.

To gain further insights into the growth of the mushy layer, we examine the spatial and temporal evolution of the mush temperature $T(z, t)$ during late modulation periods. Figure 2 shows the fluid temperature measured at various different heights for four initial fluid concentrations. The time traces of temperature show the prominent feature of periodic oscillations, with oscillation amplitudes and phases dependent on height. They demonstrate propagation of thermal oscillations with substantial decay through the mushy layer. For a quantitative analysis, we determine the amplitudes and phases of the temperature oscillation by the maxima (minima) in $T(z, t)$, marked by the red (blue) circles in Fig. 2. The temperature amplitude, $\delta T=T_{p}-T_{v}$, is given by the difference between the maximum $\left(T_{p}\right)$ and minimum $\left(T_{v}\right)$ temperature within one oscillation period. The phase of oscillation, $\delta \phi=2 \pi\left[t\left(T_{v}\right)-t\left(T_{b, v}\right)\right] / \tau$, is defined as the phase difference between $T_{v}$ and the cooling boundary temperature $T_{b}(t)$. Results of $\delta T$ and $\delta \phi$ at different mush heights are depicted in Fig. 3. We see that at late times the oscillation phase of $T(z, t)$ lags behinds $T_{b}(t)$, and $\delta \phi$ increases almost linearly with increasing mush height $z$. Thus, thermal oscillations propagate with a constant velocity through the mushy layer. The propagation speed, $v=d z / d(\delta \phi)$, is determined by the slope of the phase-lag profile $\delta \phi(z)$ and depicted as a function of the initial concentration $C_{0}$ in Fig. 4a. The oscillation amplitudes in $T(z, t)$ are shown to decrease exponentially with increasing height within the mush: $\delta T \propto e^{-\gamma z}$ (Fig. 3b). The decay rates of the amplitude $\gamma$ are determined by the profiles of $\delta T(z)$. Results of $\gamma$ for various $C_{0}$ are shown in Fig. $4 \mathrm{~b}$.

In appendix B we describe a simplified theoretical calculation with assumed constant material properties. Modulation with small amplitude $A \ll \Delta T_{0}=T_{b, 0}-T_{L}\left(C_{i}\right)$ and high frequency modulation compared to the timescale of diffusion, $\omega h_{0}^{2} / \alpha \gg 1$, produces a long 

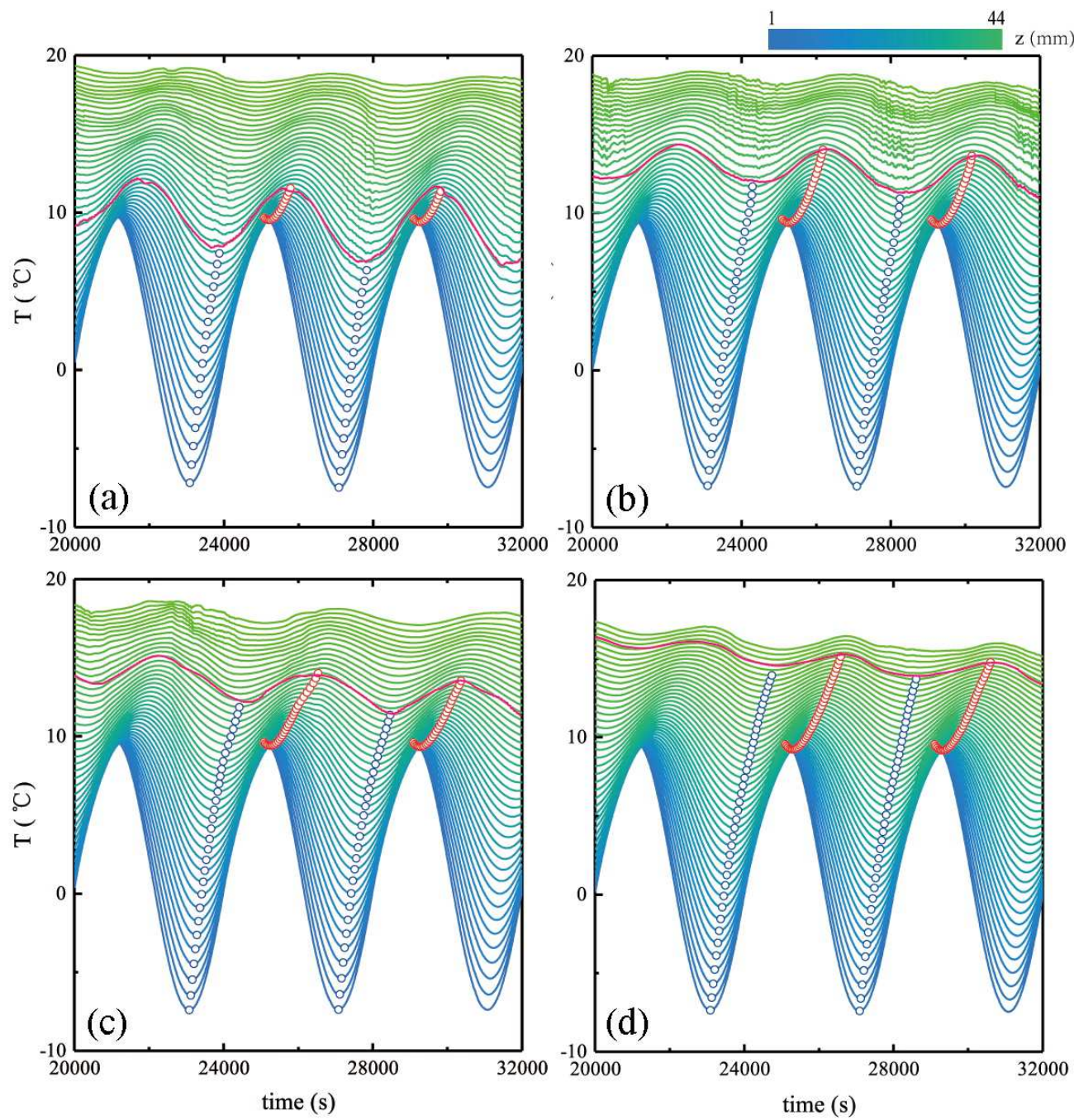

Figure 2. Time series of temperature at various heights $T(z, t)$ measured from the 5 th to the 7 th modulation period. The coloration represents the height on a linear scale, as indicated by the upper colour bar. The red curve shows the position of the mush-liquid interface. Local minima and maxima of $T(z, t)$ in the mush region are marked by blue and red circles, respectively. Results for $A=10 K, \tau=4000 \mathrm{~s}$, with initial concentration (a) $C_{0}=26 w t \%$, (b) $28 w t \%$, (c) $30 w t \%$, and (d) $32 w t \%$.

time solution

$$
T=T_{b, 0}+\Delta T_{0} \frac{z}{h_{0}}+A \mathrm{e}^{-z \sqrt{\omega / 2 \alpha}} \sin \left(\omega t-z \sqrt{\frac{\omega}{2 \alpha}}+\phi_{0}\right),
$$

where $C_{i}$ is the concentration at the mush-liquid interface, $h_{0}$ is the steady state mushy layer thickness at long times in the absence of modulation, and $\alpha$ the thermal diffusivity of the mushy layer (here assumed to be spatially uniform). The time dependent perturbation to the steady state yields a classical thermal wave solution for diffusion with periodically modulated boundary temperature (c.f. section 2.6 of Carslaw \& Jaeger 1959). This predicts a constant propagation speed $v=\sqrt{2 \alpha \omega}$ for the phase of the sinusoidal 

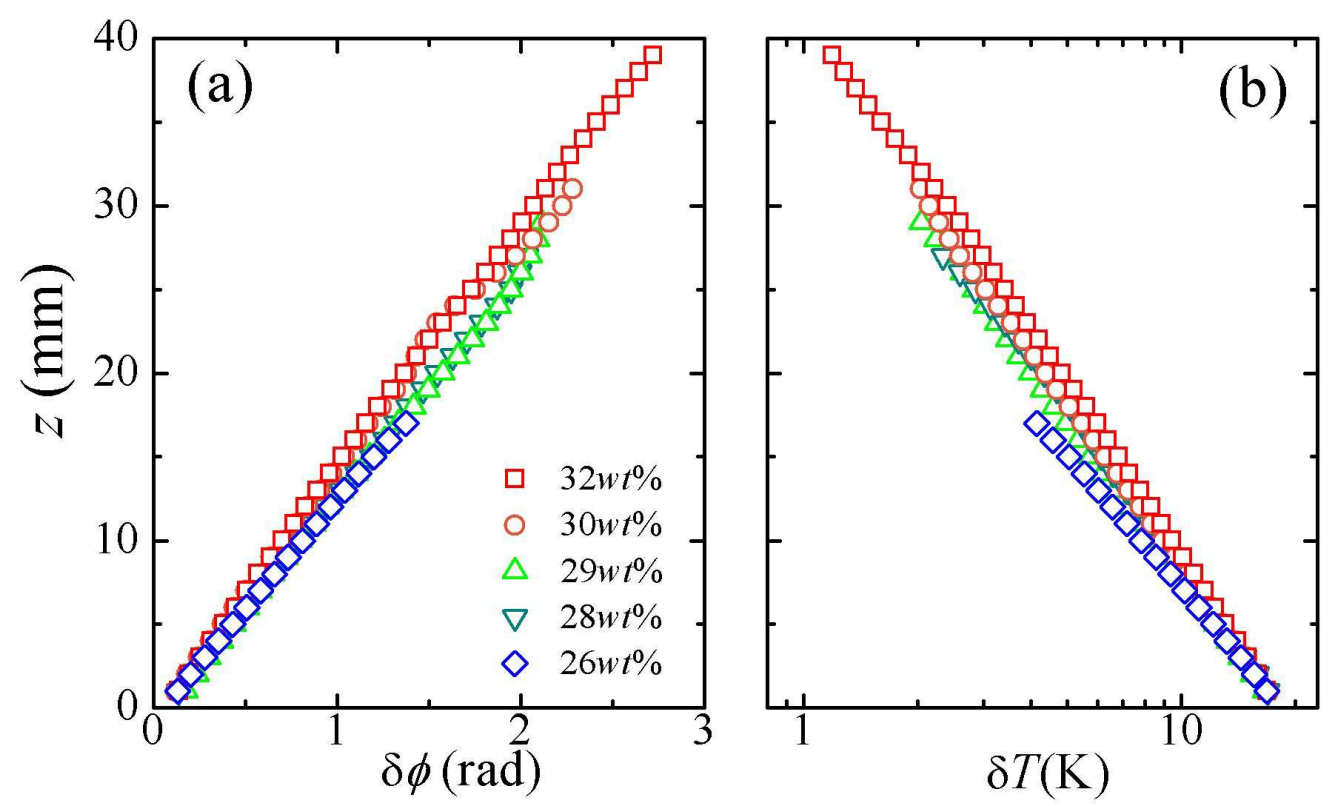

Figure 3. Properties of temperature oscillations in $T(z, t)$ as functions of the mushy-layer height for various initial concentrations. (a) The phase lags between the minima of $T(z, t)$ and the cooling boundary temperature $T_{b}(t)$. (b) The peak-to-trough temperature amplitude $\delta T=T_{p}-T_{v}$. Data are measured from the 5 th to the 6 th modulation period and extracted from the temperature traces shown in Fig. 2, for different concentrations.

oscillations of temperature (giving rise to the nomenclature of a thermal wave). It also predicts exponential decay of the oscillation amplitude with height over the lengthscale $\gamma^{-1}=\sqrt{2 \alpha / \omega}$ which corresponds to a lengthscale for diffusion over a modulation period. This captures the observed pattern of phase propagation and exponential decay of amplitude with height seen in Fig. 2 and 3. Furthermore, the theory predicts that the combination of scales $v \gamma=\omega$, which is independent of $\alpha$ and other parameters. This is in reasonable agreement with the data in Fig. 4c, where $\omega=1.57 \times 10^{-3} \mathrm{rad} \mathrm{s}^{-1}$ is constant. The agreement seems surprisingly good, given that we have neglected variation of the material properties with $z$, and that the assumption $A \ll \Delta T_{0}$ is rather marginal. Hence we conclude that the temperature evolution seen in Fig. 2 is consistent with a diffusive thermal wave superimposed on a background steady state, with the decay rate $\gamma^{-1}=\sqrt{2 \alpha / \omega}$ and phase propagation velocity $v=\sqrt{2 \alpha \omega}$ varying with an effective diffusivity $\alpha$ which depends on concentration $C_{0}$. This suggests the propagation of temperature oscillations through a mushy layer are substantially influenced by the initial fluid concentration, since the latter may significantly alter the thermal properties of the mushy layer. In general, solidification of a binary aqueous solution with a higher initial concentration results in a mushy layer with larger mean solid fraction. Based on the volume-weighted average, the mean thermal conductivity of the mushy layer $k_{m}=k_{s} \phi+k_{l}(1-\phi)$ becomes greater, and the specific heat $c p_{m}=c p_{s} \phi+c p_{l}(1-\phi)$ is less, which increases the effective thermal diffusivity $\alpha=k_{m} / c p_{m}$. (Here the thermal conductivity $k$ and heat capacity per unit volume $c p$ have subscript $s$ in the solid phase, subscript $l$ in the liquid phase, and subscript $m$ for the weighted value for the mushy layer). Thus a larger concentration leads to thermal waves with a faster propagation speed and a smaller decay rate.

Another notable feature of the mush temperature shown in Fig. 2 is that the evolution 


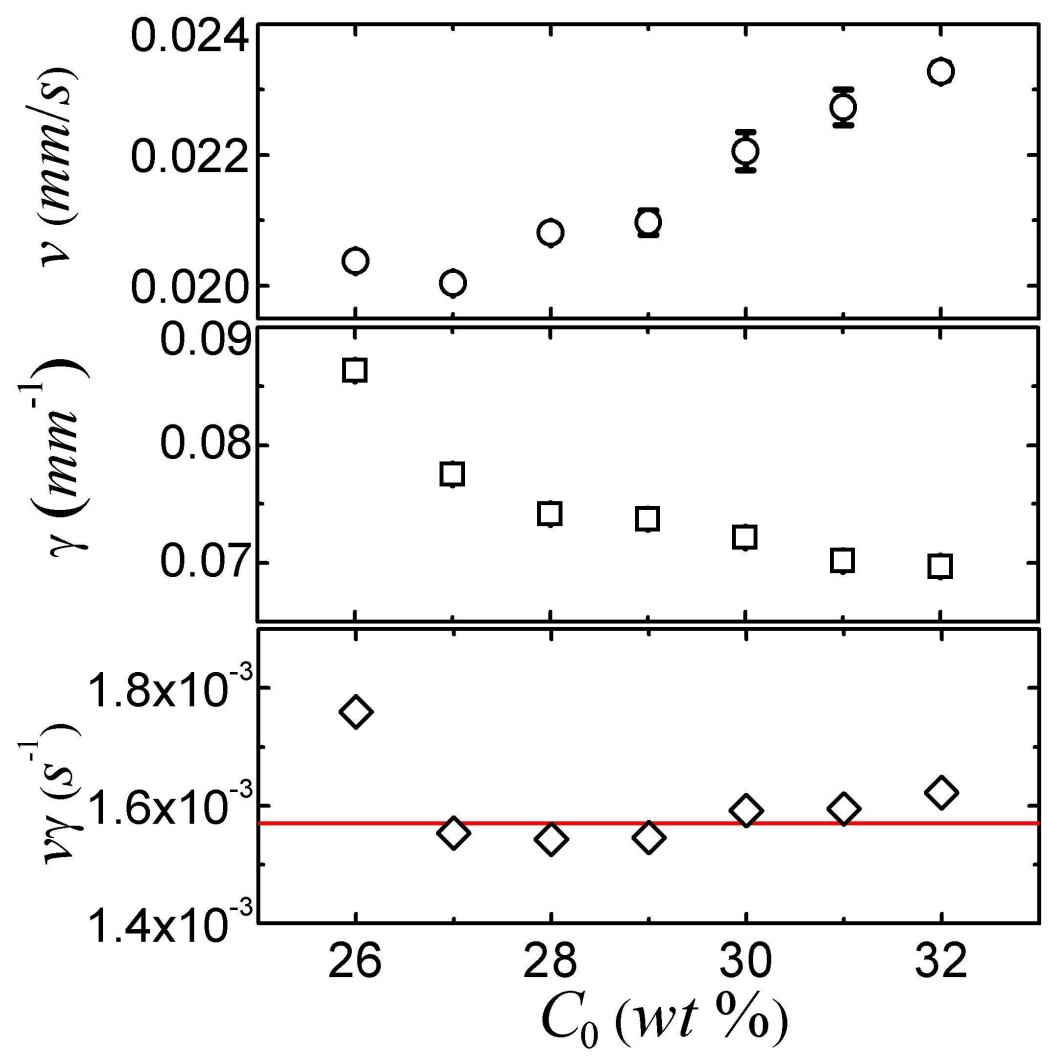

Figure 4. (a) Propagation speed of the thermal waves $v$ for different $C_{0}$, where $v$ is determined by the initial slope of the phase lag profiles $\delta \phi(z)$ shown in Fig. 3a. (b) The decay rates $\gamma$ of the amplitude of temperature oscillations for different $C_{0}$ calculated from Fig. 3b. (c) Product of $v \gamma$ as a function of $C_{0}$. The red line shown the theoretical prediction $v \gamma=\omega=1.57 \times 10^{-3} \mathrm{rad} / \mathrm{s}$.

of $T(z, t)$ during one modulation period becomes highly repeatable at each height near the bottom cooling boundary. For a given $z$, oscillations of the mush temperature are very close to periodic, with nearly identical amplitudes and phases in different modulation periods. The well-repeated temperature responses in the mushy layer to the applied thermal modulation indicate that there is no significant trend in the variation of local thermal properties in the mush over time. This is suggestive of either an approximately time-independent porosity structure in the mush region, particularly near the bottom cooling boundary, or else one that oscillates periodically in time. Such a stationary mush structure appears in the late growth periods when convection in the interstitial melt diminishes and the mushy layer becomes stagnant. This limits changes to the bulk concentration of the ice via gravity drainage, and creates an appropriate condition for studying the distributions of solid fraction through temperature measurements (see §4).

For a direct comparison of the temperature distribution in the mushy layers that grow from different initial concentrations, we present in Fig. 5 normalized vertical temperature profiles $T^{*}\left(z^{*}\right)$ measured at four moments $(t=i / 4 \tau, i=1,2,3,4)$. Here the mush temperature is nondimensionalized, $T^{*}(t)=\left(T(z, t)-T_{b, 0}\right) /\left(T_{i}(t)-T_{b, 0}\right)=T(z, t) / T_{i}(t)$, with the mean cooling boundary temperature being $T_{b, 0}=0^{\circ} C$ and $T_{i}(t)$ the temperature at the mush-liquid interface. The height is normalized by the mushy-layer thickness $z^{*}(t)=z / h(t)$. Results for both $C_{0}=26 w t \%$ and $29 w t \%$ imply that variations of the 


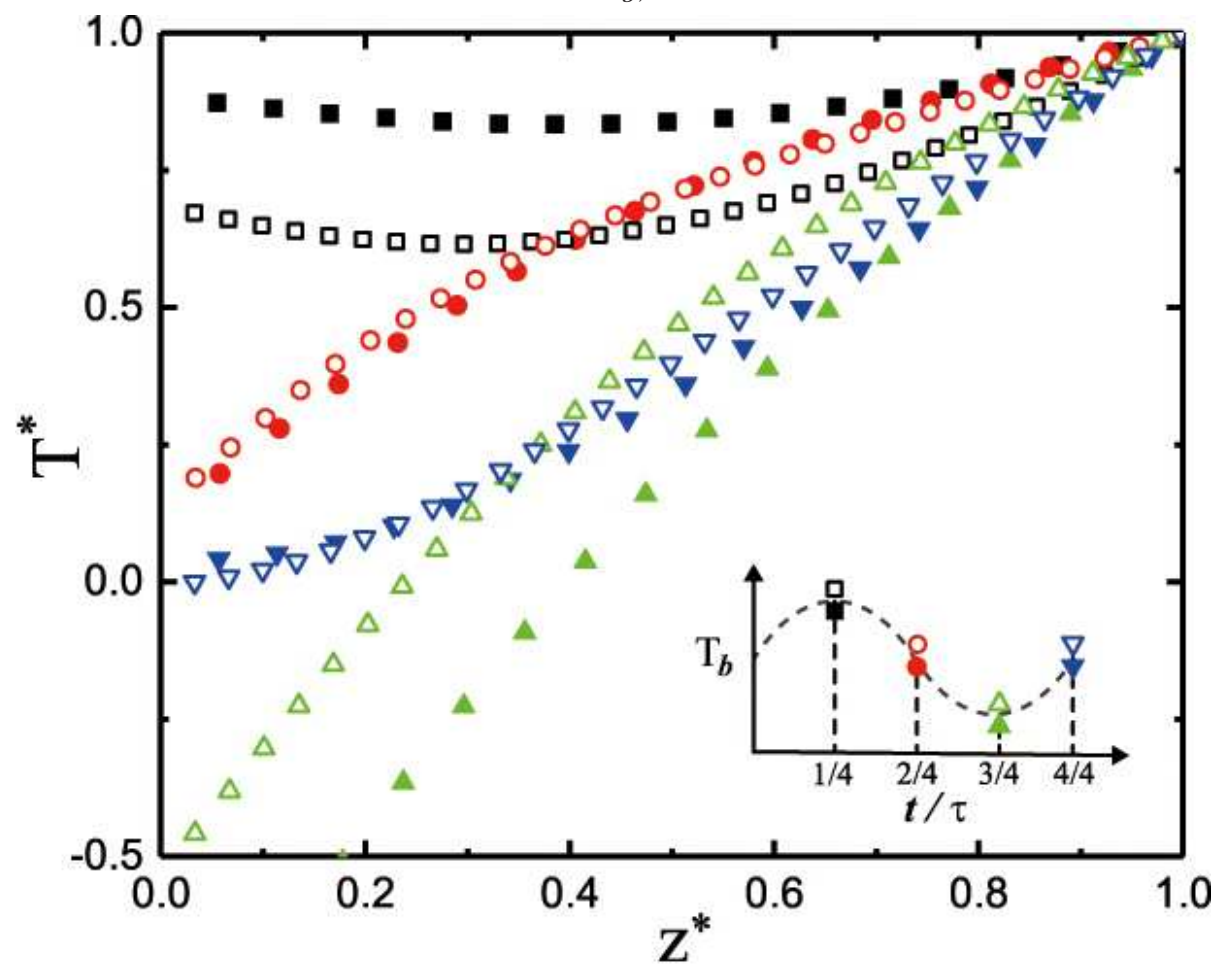

Figure 5. Normalised vertical temperature profiles of the mushy layer $T^{*}\left(z^{*}\right)=T / T_{i}(t)$ as a function of $z^{*}(t)=z / h(t)$ measured at four phases during the 6th modulation period. Solid symbols: data for $C_{0}=26 w t \%$; Open symbols: data for $C_{0}=29 w t \%$. The inset sketches the variation of the bottom temperature in these four phases. Results for $A=10 K, \tau=4000 \mathrm{~s}$.

mush-temperature profiles can be categorized into two phases that depend on variations of cooling boundary temperature $T_{b}$. In a decreasing phase $(\tau / 4 \leqslant t<3 \tau / 4)$ when $T_{b}$ is decreasing in time, the slope of $T^{*}\left(z^{*}\right)$ decreases with increasing height. Thus curves of $T^{*}\left(z^{*}\right)$ in this phase are convex. However, when $T_{b}$ is increasing $(0 \leqslant t<\tau / 4$ and $3 \tau / 4 \leqslant t<\tau), T^{*}\left(z^{*}\right)$ is concave with slope increasing with $z^{*}$. As discussed above, such a depth-dependence of the mush temperature is due to the finite thermal diffusivity that causes time delays when the bottom-temperature oscillations diffuse through the mushy layer, with the phase lag of $T(z, t)$ behind $T_{b}(t)$ increasing when the height increases (see Fig. 3a).

It is worth noting that a negative slope of $T^{*}\left(z^{*}\right)$ appears at small mush heights when $T_{b}$ is near a maximum $(t=\tau / 4)$. A negative temperature gradient in the mushy layer is indicated as well in Fig. 2 near the maxima of $T(z, t)$ at low heights (where the time traces for different heights cross). We attribute this phenomenon to the mush temperature varying more rapidly near the base due to thermal modulations than the slower diffusive propagation of the temperature oscillations through the mushy layer to greater heights. This can be rationalised using the approximate thermal wave solution (3.1). Setting $\phi_{0}=0$ and letting $\gamma=\sqrt{\omega / 2 \alpha}$, then from (3.1) the temperature gradient in the mushy layer is

$$
\frac{\partial T}{\partial z}=\frac{\Delta T_{0}}{h_{0}}-\gamma A \mathrm{e}^{-\gamma z}[\sin (\omega t-\gamma z)+\cos (\omega t-\gamma z)]
$$


For a given depth $z$, this has a minimum value $\Delta T_{0} / h_{0}-\sqrt{2} \gamma A \mathrm{e}^{-\gamma z}$ at times $\omega t-\gamma z=$ $\pi / 4+2 n \pi$ for integers $n$. For large enough values of $\gamma A$, the temperature gradient $\partial T / \partial z$ will be negative for small $z$ during an interval of time about $\omega t-\gamma z \approx \pi / 4+2 n \pi$. For example, for $C_{0}=29 \mathrm{wt} \%$, Fig. $4 \mathrm{~b}$ gives $\gamma=0.074 \mathrm{~mm}^{-1}$ whilst $A=10 \mathrm{~K}$ in Fig. 5, yielding $\gamma A \sqrt{2} \approx 1.0 \mathrm{~K} \mathrm{~mm}^{-1}$, whilst $\Delta T_{0} / h_{0} \approx 0.5 \mathrm{~K} \mathrm{~mm}^{-1}$. Hence, the thermal wave solution predicts that $\partial T /\left.\partial z\right|_{z=0}$ will be negative for a period of time centered around $t=\tau / 8$ into the modulation period. Note that periods with $\partial T /\left.\partial z\right|_{z=0}<0$ will have a positive heat flux from the cooling plate into the mushy layer, despite the overall trend for cooling by the lower boundary. We further expect that this effect is more pronounced for low initial concentrations, when the background temperature gradient is smaller (c.f. Fig. 2), and in experimental conditions with large modulation amplitude and frequency, when the thermal wave component dominates the variation of the mush temperature.

Results in Fig. 5 also suggest that the mush temperature profiles are dependent on the initial fluid concentration. Large deviations in $T^{*}\left(z^{*}\right)$ are seen from the profiles with different $C_{0}$ when $T_{b}$ reaches a maximum $(t=\tau / 4)$ or a minimum $(t=3 \tau / 4)$. For a lower $C_{0}$ the temperature span crossing the mushy layer $T_{i}-T_{b}$ is smaller due to the lower liquidus temperature $T_{i}=T_{L}\left(C_{i}\right)$, and hence there is a larger oscillation amplitude in the dimensionless temperature $T^{*}$. When $T_{b}=0^{\circ} \mathrm{C}(t=2 \tau / 4,4 \tau / 4)$ profiles of $T^{*}\left(z^{*}\right)$ for the two concentrations appear closer to each other. However, there are still notable differences between the two curves in these phases, indicative of differing thermal properties of the mushy layer due to the differing fluid concentrations. Finally we note that the variations of the profiles $T^{*}\left(z^{*}\right)$ within one modulation cycle shown in Fig. 5 are typical and repeatable in different modulation periods (c.f. Fig. 2).

\section{Inferring the solid-fraction distribution in mushy layers}

\subsection{Heat transports within a mushy layer}

In growing mushy layers, heat transport between the mush and the liquid region occurs mainly through two types of processes. At early times, convection mainly occurred at the mush-liquid interface in the form of small-scale salt fingers in the presence of a stabilizing thermal gradient. This boundary-mode convection is driven by the compositional gradient that is established due to the formation of a solute-depleted boundary layer ahead of the growing mushy layer. As the mushy layer grows and reaches a critical height, a second type of convection occurs as a result of a hydrodynamic instability due to density gradients within the mushy-layer (Worster 1992). In this mushy-layer mode convection, large-scale plumes emanate from solid-free chimneys dissolved in the interior of the mushy layer, in a process often called gravity drainage. Heat and solute transport through the mushylayer mode convection plays an important role in determining the heat budget of growing mushy layers. During late growth periods, however, mushy-layer convection approaches an asymptotic state when the growth rate approaches zero (Tait \& Jaupart 1989). The time-integrated expulsion of light solution modifies the concentration in the liquid region and causes the liquid concentration $C_{l}$ just above the mushy layer to decrease. In the following we will demonstrate that convection driven by the buoyancy force within the mushy layer carries a negligible heat transport compared to thermal conduction within the mushy layer.

The governing equation of the temperature distribution $T(z, t)$ within a mushy layer 


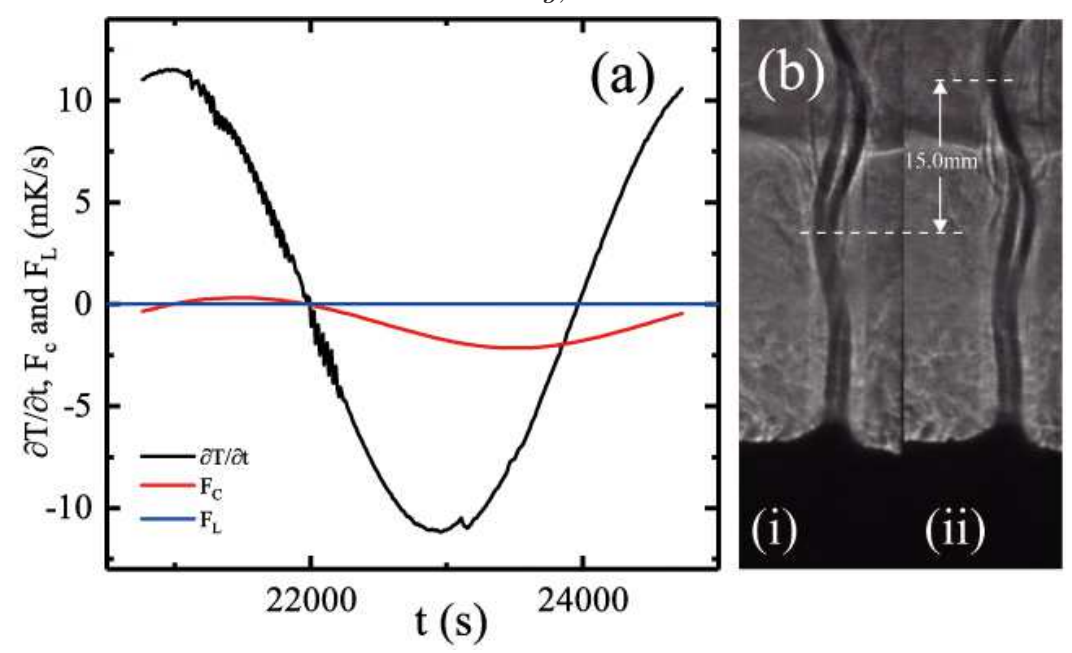

Figure 6. (a) Components of the heat equation estimated at the mid-height $(z=12 \mathrm{~mm})$ of the mushy layer. Here the latent heating contirbution $F_{L}=1.3 \times 10^{-2} \mathrm{mK} / \mathrm{s}$ is determined by the difference of $\langle\phi\rangle$ measured in the 5th and 6th modulation period, whilst the convective contribution $F_{c}$ is discussed further in the text. (b) Shadowgraph images illustrate the dynamical features of the plume flows from chimneys that undergo coiling instability. Results for an initial concentration $C_{0}=29 w t \%, A=10 K$ and $\tau=4000$ s.

reads

$$
\frac{\partial T}{\partial t}+\underbrace{\frac{c p_{l}}{c p_{m}} w \frac{\partial T}{\partial z}}_{F_{c}}=\underbrace{\frac{1}{c p_{m}} \frac{\partial}{\partial z}\left(k_{m} \frac{\partial T}{\partial z}\right)}_{F_{d}}+\underbrace{\frac{L}{c p_{m}} \frac{\partial \phi}{\partial t}}_{F_{L}},
$$

relevant to a vertical measurement profile located away from chimneys. The physical parameters are $c p$ the specific heat, $k$ the thermal conductivity and $L$ the latent heat. The subscript $m$ and $l$ indicate the quantities in the mush and pure liquid region, respectively. The convective Darcy velocity $w$ arises in the mushy layer due to the gravity drainage. Previous theoretical and numerical modelling work suggests that significant horizontal thermal gradients occur in a narrow boundary layer near each chimney due to the cooling effect of cold solute depleted plumes. However, the temperature gradient is predominantly vertical in the mushy layer further away from the chimneys (Rees Jones \& Worster 2013; Wells et al. 2010). We have hence neglected horizontal temperature gradients for profiles taken outside of the chimneys and their associated thermal boundary layers. This heat equation expresses that temperature evolution in a mushy layer, $\partial T / \partial t$, depends on heattransport components including thermal advection $F_{c}$, thermal conduction $F_{d}$ and the latent heat flux $F_{L}$ (all normalised by $c p_{m}$ ). In the following, we estimate the magnitude of the convective heat transport $F_{c}$ and the latent heating $F_{L}$, and their contribution to the heat budget of the system.

In order to estimate the convective heat transfer $F_{c}$ in a mushy layer, we estimate the fluid velocity of the large-scale plume flows from the chimneys. Figure $6 \mathrm{~b}$ presents two snapshots of the plumes emanating from chimneys in the interior of the mushy layer, in which the upward wavy motion of the plumes is clearly seen. Assuming that the waves are generated by a generalization of the Kelvin-Helmholtz instability (following similar analysis of saline plumes, Pesci et al. 2003), the vertical fluid velocity $W$ within the chimney plume can be determined through $(W-u) / 2=c$, where $c$ is the propagation speed of the wave and $u$ is the vertical velocity in the background fluid surrounding the plume 


\section{Quantity}

Specific heat of crystal per unit volume Specific heat of solution per unit volume

Thermal conductivity of crystal

Thermal conductivity of solution

$\begin{array}{ccc}\text { Symbol } & \text { Value } & \text { Unit } \\ c p_{s} & 2.28 \times 10^{6} & \mathrm{~J} \mathrm{~m}^{-3} \mathrm{~K}^{-1} \\ c p_{l} & 3.68 \times 10^{6} & \mathrm{~J} \mathrm{~m}^{-3} \mathrm{~K}^{-1} \\ k_{s} & 2.2 & \mathrm{~J} \mathrm{~m}^{-1} \mathrm{~s}^{-1} \mathrm{~K}^{-1} \\ k_{l} & 0.54 & \mathrm{~J} \mathrm{~m}^{-1} \mathrm{~s}^{-1} \mathrm{~K}^{-1}\end{array}$

TABLE 1. The parameter values used in calculation (Peppin et al. 2008).

(with $u \ll W)$. Using the time interval $\Delta t=5.3 \mathrm{~s}$ between the two pictures, and the vertical displacement $\Delta z=15 \mathrm{~mm}$ that the left crest of the wavy plume has made, we obtain $c=\Delta z / \Delta t=2.8 \mathrm{~mm} \mathrm{~s}^{-1}$ and $W=2 c+u \approx 2 c=5.6 \mathrm{~mm} \mathrm{~s}^{-1}$. The mean diameter of the chimney $d=0.8 \mathrm{~mm}$, and the mean spacing between two chimneys in this period $D=40 \mathrm{~mm}$, are determined using a large amount of statistical data from the top-view images of the mushy layer. Assuming that the upward volume flux from the mush to the liquid region is mainly driven by the plumes, through mass conservation we obtain the mean downward vertical velocity through the mush liquid interface $\langle w\rangle \approx \pi d^{2} W /(2 D)^{2}=1.8 \times 10^{-3} \mathrm{~mm} \mathrm{~s}^{-1}$, where we have assumed that each channel has circular cross section and lies within a square cross sectional cell.

In Fig. 6 a we compared the magnitudes of $\partial T / \partial t$ with the convective heat transport, $F_{c}$, and the latent heating, $F_{L}$ (where the latter two are rescaled by $c p_{m}$ ). Here the mean trend in $F_{L}=1.3 \times 10^{-2} \mathrm{mK} / \mathrm{s}$ is determined by the difference of $\langle\phi\rangle$ using the concentration data measured in the liquid region during the 5 th and 6 th modulation period, following the experimental procedure discussed in section 4.4. The results imply that in the late modulation periods the contribution to the heat equation from the trend in latent heating is negligible, since the mushy layer barely grows and the mean internal solid fraction hardly changes over a modulation period. In appendix A we consider the potential magnitude of periodic latent heating and cooling due to periodic oscillations in $\phi(z, t)$. This effect is fairly modest, and is neglected below. Over the whole modulation period, heat transport by convection is a lot smaller than the rate of change of the specific heat. According to (4.1), heat transport through the mushy layer is mainly by thermal conduction. Such a weak-convection simplification is appropriate in late growth periods. In the following section we will compute various components in the heat equation using the temperature data $T(z, t)$ measured inside the mushy layer, and determine the solid fraction distribution $\phi(z)$ of the mushy layer.

\subsection{Theoretical approach in analyzing the temperature data}

We have measured the heat-transfer terms and shown that when the mushy layer grows in an asymptotic state, the advection term does not play an important role in the heat transport equation (4.1). Furthermore, the porosity structure of the mushy layer shows no long term trends over time as revealed by the temperature distribution data (Fig. 2), and the corresponding latent heat flux is negligible (see Fig. 6). We therefore approximate $\phi(z)$ and the resulting local mush properties (such as $k$ and $c p$ ) by their time-averaged values over the time scale of each modulation period. The potential impact of neglecting time-periodic variations in $\phi(z, t)$ is discussed in appendix $\mathrm{A}$, where we estimate that neglecting such variations results in an error of less than 0.1 in estimates of $\phi$. With all these considerations, the heat transport equation in the mushy layer is reduced to a 

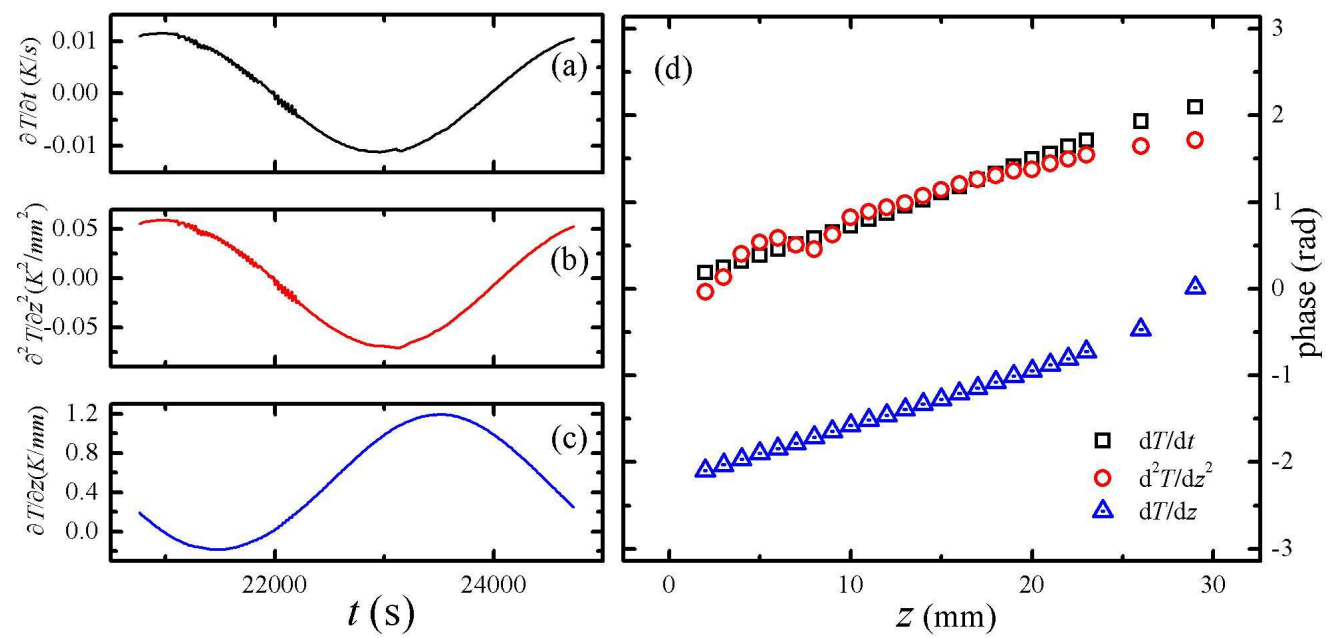

Figure 7. (a-c) Results for $\partial T / \partial t, \partial^{2} T / \partial z^{2}$ and $\partial T / \partial z$ as a function of time measured during the 6th modulation period at $z=12 \mathrm{~mm}$. (d) Phases for $\partial T / \partial t, \partial^{2} T / \partial z^{2}$ and $\partial T / \partial z$ measured at different heights. Results for an initial concentration $C_{0}=29 w t \%, A=10 \mathrm{~K}$ and $\tau=4000 \mathrm{~s}$.

one-dimensional conduction equation

$$
\underbrace{\frac{\partial T}{\partial t}}_{F_{t}}=\alpha_{m} \frac{\partial^{2} T}{\partial z^{2}}+\underbrace{\frac{1}{c p_{m}} \frac{\partial k_{m}}{\partial z} \frac{\partial T}{\partial z}}_{F_{\alpha}},
$$

where $\alpha_{m}=k_{m} / c p_{m}$ is the thermal diffusivity. All of the temperature derivatives are calculated from the experimental data $T(z, t)$ as follows. We apply the first order forward scheme to calculate the first derivative of $T(z, t)$. A cubic smoothing spline is used to filter out the noise in $\partial T / \partial z$ due to local temperature fluctuations. Then the first order backward scheme is applied to the results of $\partial T / \partial z$ to evaluate the second order derivative $\partial^{2} T / \partial z^{2}$. The thermal diffusivity $\alpha_{m}$, specific heat $c p_{m}$ and thermal conductivity $k_{m}$ are functions of the solid fraction $\phi$, and thus (4.2) reduces to an equation that can be solved for $\phi$ at each height $z$.

We examine the temporal properties of each term in equation (4.2). Figure 7 presents $\partial T / \partial t, \partial^{2} T / \partial z^{2}$ and $\partial T / \partial z$ as functions of time at a given height $z=12 \mathrm{~mm}$. We see that both the temporal and spatial derivatives varied periodically in time in response to the modulation of $T_{b}$. If one assumes that $\phi$ and hence the material properties evolve much slower than the rapid variations of temperature, then the phases of $F_{T}$ and $F_{\alpha}$ are given by the phases of $\partial T / \partial t$ and $\partial T / \partial z$, respectively. Moreover, the phases of both sides of equation (4.2) must be the same.

A first glance at figure 7 suggests that the phases of $\partial^{2} T / \partial z^{2}$ and $\partial T / \partial t$ are close to each other. Thus the magnitude of $F_{\alpha}$ should be relatively small. However, closer inspection of the data reveals that there are still slight phase differences between $\partial^{2} T / \partial z^{2}$ and $\partial T / \partial t$. Figure 8 a shows $\partial^{2} T / \partial z^{2}$ against $\partial T / \partial t$ measured at different heights. If $\partial^{2} T / \partial z^{2}$ and $\partial T / \partial t$ have the same phase, then data points in the figure should collapse into a straight line with $\partial T / \partial t \propto \partial^{2} T / \partial z^{2}$. However, one can see that the data points in fact constitute a curve with the shape of a tilted ellipse, indicating there is a nontrivial difference in phase between $F_{T}$ and $F_{\alpha}$ and thus the first-derivative term $F_{\alpha}$ is non-negligible.

To account for the effect of the periodically varying mush temperature and the fact 


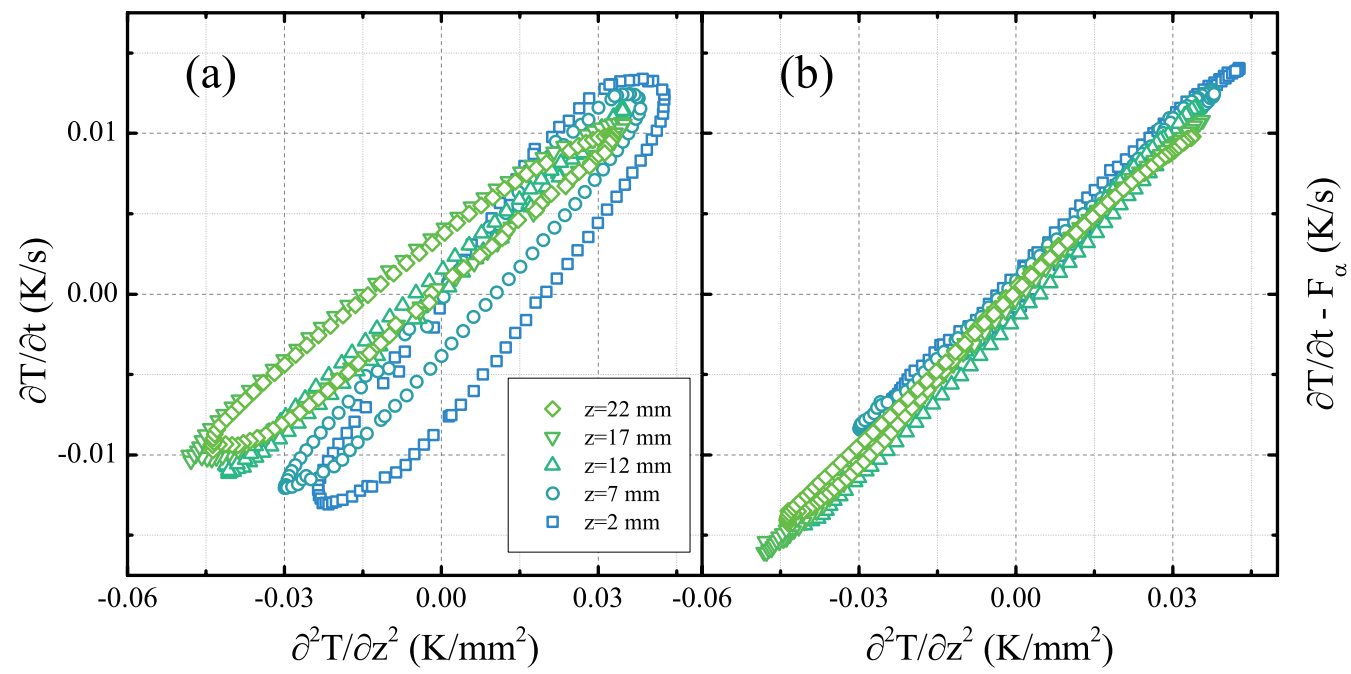

Figure 8. Heat-flux diagrams. (a) $\partial T / \partial t$ as a function of $\partial^{2} T / \partial z^{2}$, and (b) $\partial T / \partial t-F_{\alpha}$ as a function of $\partial^{2} T / \partial z^{2}$. Results for an initial concentration $C_{0}=29 w t \%, A=10 \mathrm{~K}$ and $\tau=4000 \mathrm{~s}$.

that $F_{T}$ dominates the conductive heat transport, we adopt a mathematical scheme to resolve $\alpha_{m}$ from the heat equation. We rewrite (4.2) in the following form:

$$
\alpha_{m} \frac{\partial^{2} T}{\partial z^{2}}=\frac{\partial T}{\partial t}-p_{m} \frac{\partial T}{\partial z}
$$

where $p_{m}=1 / c p_{m}\left(\partial k_{m} / \partial z\right)$. We first determine $p_{m}$ at each depth, using the fact that the phase of $\partial T / \partial t-p_{m} \partial T / \partial z$ should be identical to that of $\partial^{2} T / \partial z^{2}$. The calculation of $p_{m}$ is achieved through a shooting scheme, with the value of $p_{m}$ initially given and adjusted according to the phase difference between $\partial T / \partial t-p_{m} \partial T / \partial z$ and $\partial^{2} T / \partial z^{2}$. Having determined $p_{m}$, we then solve for the thermal diffusivity $\alpha_{m}$ given by the constraint that the magnitudes of both sides in (4.2) have to be the same. With $p_{m}$ determined through this scheme, we plot $\partial T / \partial t-F_{\alpha}$ as a function of $\partial^{2} T / \partial z^{2}$ in Fig. 8b. Data points in Fig. 8b collapse into straight lines crossing the origin. The thermal diffusivity $\alpha_{m}$ is then determined by linear fitting of the data using a least-square method. We find results of $\alpha_{m}(z)$ are nearly independent of the modulation period, and thus validates the assumption that $\phi$ has no long term trend over time and is insensitive to the rapid variations of temperature (see also results in Fig. 10). Profiles of the solid fraction $\phi$ are finally determined from $\alpha_{m}(z)=k_{m} / c p_{m}$ where $k_{m}$ and $c p_{m}$ are expressed in terms of $\phi$ using the physical parameters shown in table 1 . Note that the collapse seen in Fig. $8 \mathrm{~b}$ shows some similarities with the method used by Pringle et al. (2007) to determine the thermal conductivity of sea ice from a fit of $k_{m} \partial^{2} T / \partial z^{2}$ versus $\rho \partial U / \partial t-\left(\partial k_{m} / \partial z\right)(\partial T / \partial z)$ where $U$ is the sea ice internal energy per unit mass and $\rho$ the density. However, in order to determine the internal energy the approach of Pringle et al. (2007) used independent measurements of bulk concentration as a function of depth from sea ice cores along with the temperature, whilst we infer $\phi$ without independent knowledge of the bulk concentration.

In our numerical scheme $\alpha_{m}$ and $p_{m}$ are determined independently, based on the equalities of the phases and magnitudes on both sides of (4.3). Since

$$
p_{m}(z)=\frac{1}{c p_{m}} \frac{\partial k_{m}}{\partial z}=\frac{k_{s}-k_{l}}{c p_{s} \phi+c p_{l}(1-\phi)} \frac{d \phi}{d z},
$$



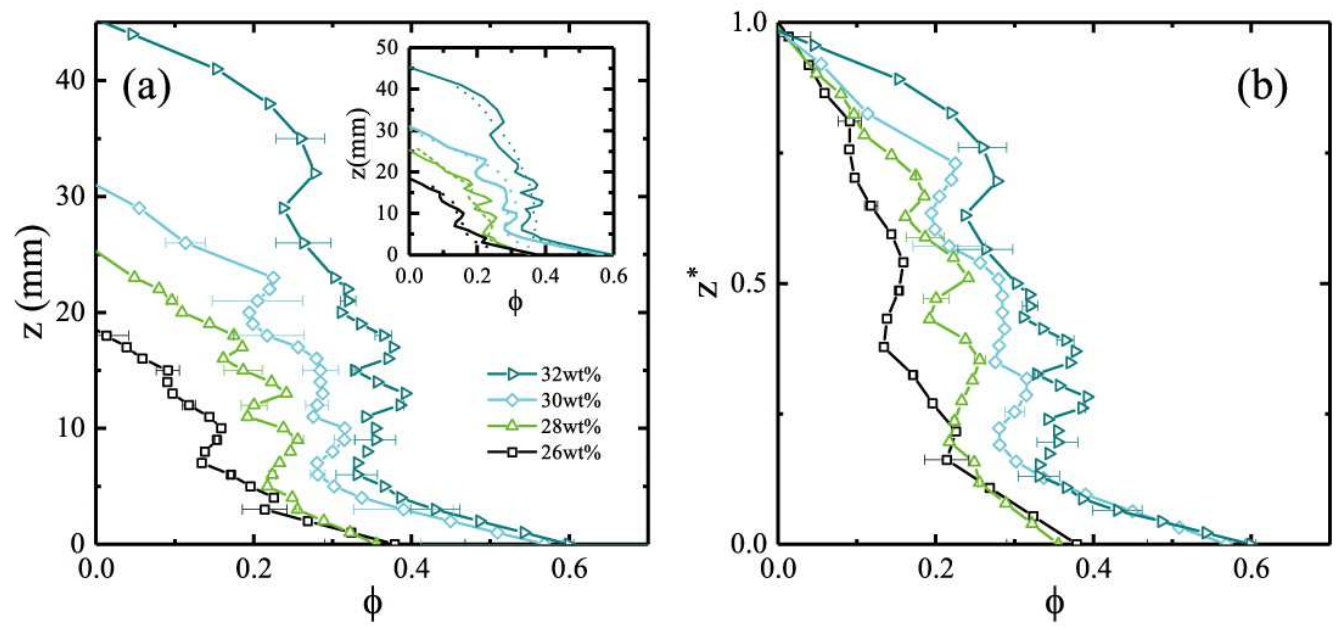

Figure 9. Profiles of $\phi(z)$ for different initial concentrations. (a) The solid fraction $\phi$ as a function of height $z$ within the mushy-layer. (b) The solid fraction $\phi$ as a function of the normalized height $z^{*}=z / h$. The error bars indicate the difference between $\phi$ and $\phi_{p}$ determined from (4.3). Inset in (a): a comparison of $\phi$ (solid line) and $\phi_{p}$ (dashed line) obtained from the separate approaches. Measurements are taken in the 6 th modulation period with $A=10 K$ and $\tau=4000 \mathrm{~s}$.

a second estimate of the solid fraction, represented by $\phi_{p}(z)$, can be obtained given by the profiles of $p_{m}(z)$. We solved (4.4) using a 4th order Runge Kutta method, integrating from the mush-liquid interface to the bottom. To reduce the error caused by the difference of height between the thermistors and the mush-liquid interface, we have employed a shooting method scheme, with the initial solid fraction $\phi_{i}$ (at the uppermost thermistor in the mush) adjusted to minimize the difference between $\overline{\phi_{p}}$ and $\bar{\phi}$. Comparing $\phi_{p}$ with $\phi$ provides a consistency check on the method.

\subsection{Distributions of solid fraction in mushy layers with various initial concentrations}

Figure 9a presents results of solid fraction profiles $\phi(z)$ for different initial concentration $C_{0}$. The mushy-layer height as well as the mean solid fraction $\bar{\phi}$ appear to increase as $C_{0}$ increases. The general features of the profiles $\phi(z)$ also depend on $C_{0}$. For a high initial concentration $C_{0}$, the solid fraction $\phi(z)$ exhibits a cliff-ramp-cliff structure: near the bottom and interface $\phi(z)$ decreases rapidly as $z$ increases; but in the bulk of the mushy-layer, $\phi(z)$ varies relatively slowly with $z$. The cliff-ramp-cliff structure is more pronounced for higher $C_{0}$. Larger local fluctuations of $\phi$ with height are also seen for higher $C_{0}$. These results suggest that as the mushy layer thickness increases for higher initial concentration, the internal dendritic structure of mushy layer becomes more complex and varies more erratically in space. On the other hand, for low $C_{0}$ the solid fraction $\phi(z)$ decreases almost linearly with $z$ with smaller fluctuations at each height, indicating the dendritic structure of the mushy layer is relatively simple. The dependence of the solid fraction profiles on the initial concentration is further demonstrated in Fig. $9 \mathrm{~b}$, where we show $\phi$ as a function of the normalized height $z^{*}=z / h$. We speculate that such variability in $\phi$ might result from prior local solute fluxes caused by gravity drainage, although this is not conclusively demonstrated here. In the inset of Fig. 9a, we compare results of $\phi(z)$ and $\phi_{p}(z)$ that were derived from $\alpha_{m}(z)$ and $p_{m}(z)$. The spatial fluctuation of $\phi$ is in general larger than that of $\phi_{p}$. Fluctuations are likely smoothed by the method used to determine $\phi_{p}$, because the process of numerical integration acts as a natural 

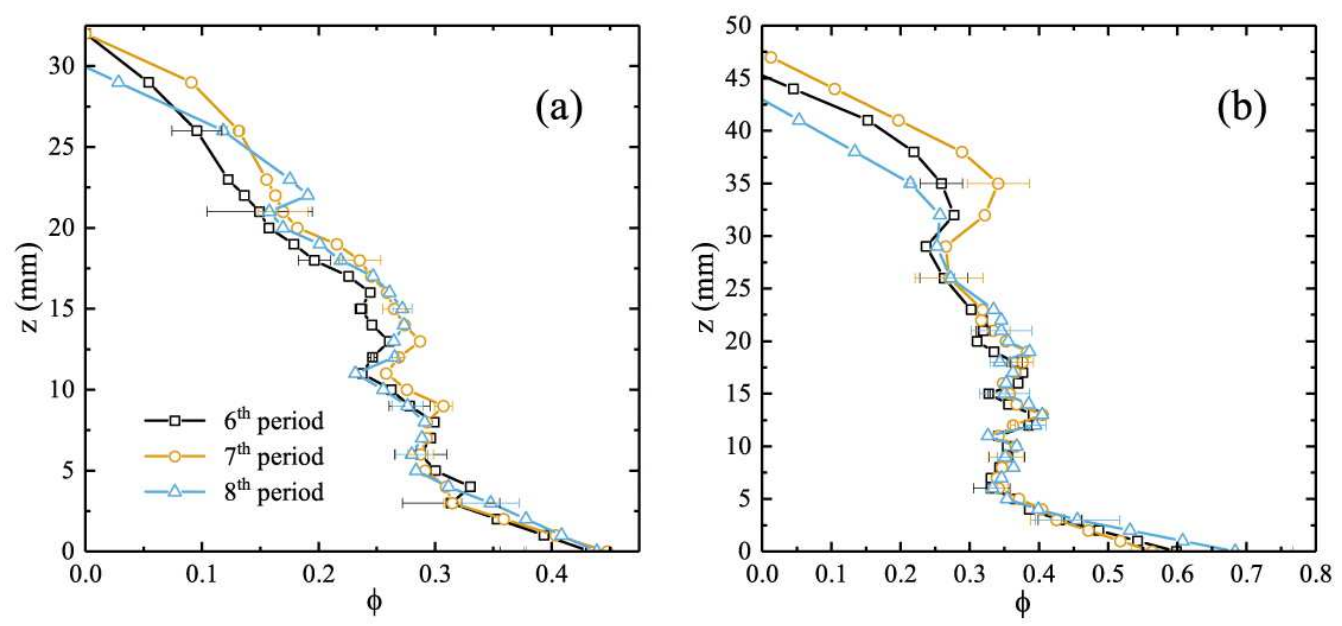

Figure 10. Profiles of $\phi(z)$ measured at different modulation periods. Results for (a) $C_{0}=29.0 w t \%$, (b) $C_{0}=32.0 w t \%$ and $A=10 K, \tau=4000 \mathrm{~s}$. The error bars indicate the difference between $\phi$ and $\phi_{p}$ determined from (5.3).

smoothing filter for high frequency fluctuations. Beyond the fluctuations, the pattern of depth-dependence of $\phi$ and $\phi_{p}$ are mainly in consistency. The deviation of $\phi_{p}$ from $\phi$ is greater when $C_{0}$ increases, indicating the greater spatial variance of the mushy-layer structure for higher $C_{0}$.

In Fig. 10 we compare results of $\phi(z)$ measured at different modulation periods $(t=6 \tau, 7 \tau$ and $8 \tau)$ with two initial concentrations $C_{0}=29.0 w t \%$ and $32.0 w t \%$. The error bar represents the difference between $\phi$ and $\phi_{p}$. We see that the solid fraction $\phi(z)$ becomes approximately independent of time throughout much of the depth in these late modulation periods, as the three profiles nearly overlap with each other (the other experiments not shown in Fig. 10 also have a similar level of time-independence of $\phi(z)$ for $t \geqslant 7 \tau$ ). Their consistency is better near the cooling boundary than that close to the mush-liquid interface. These results suggest that, in the late time period, growth of the mushy-layer is already in the asymptotic state in which both thickness and the dendritic structure of the mushy layer show only a weak long-term temporal trend. Whilst there may be some fluxes of comparatively fresh water from selected chimneys which may cause some change in bulk concentration and drift in the solid fraction, we conclude that the resulting changes to $\phi$ are small in the interior of the mushy layer over this timescale (6th to 8 th modulation periods). It is possible that the slight retreat of the mean position of the mush liquid interface (as seen in Fig. 2d) is linked to the greater variation in $\phi$ seen near the mush-liquid interface. However, the fluid advection and the turbulent heat-transport are notable close to the mush-liquid interface, and may give rise to large temperature fluctuations there. Hence our measurements of $\phi(z)$ based on the local fluid temperature data may be less repeatable very close to the mush-liquid interface. It is worth noting that results for the local fluctuations of $\phi(z)$ in the mid-height region $(5 \mathrm{~mm} \leqslant z \leqslant 20 \mathrm{~mm})$ are in close agreement in the three runs. Thus they may reveal the fine structures in the solid fraction profiles: there are large excursions of the mush porosity along the vertical profiles when growing with high initial concentrations.

The cliff-ramp-cliff structure of $\phi(z)$ measured here was also observed by Chen (1995) through computed tomography, and by Aussillous et al. (2006) using magnetic resonance imaging. It seems not to agree with the measurements of Shirtcliffe et al. (1991) and Worster (1992). The reason for this disagreement may be that the present study measured 

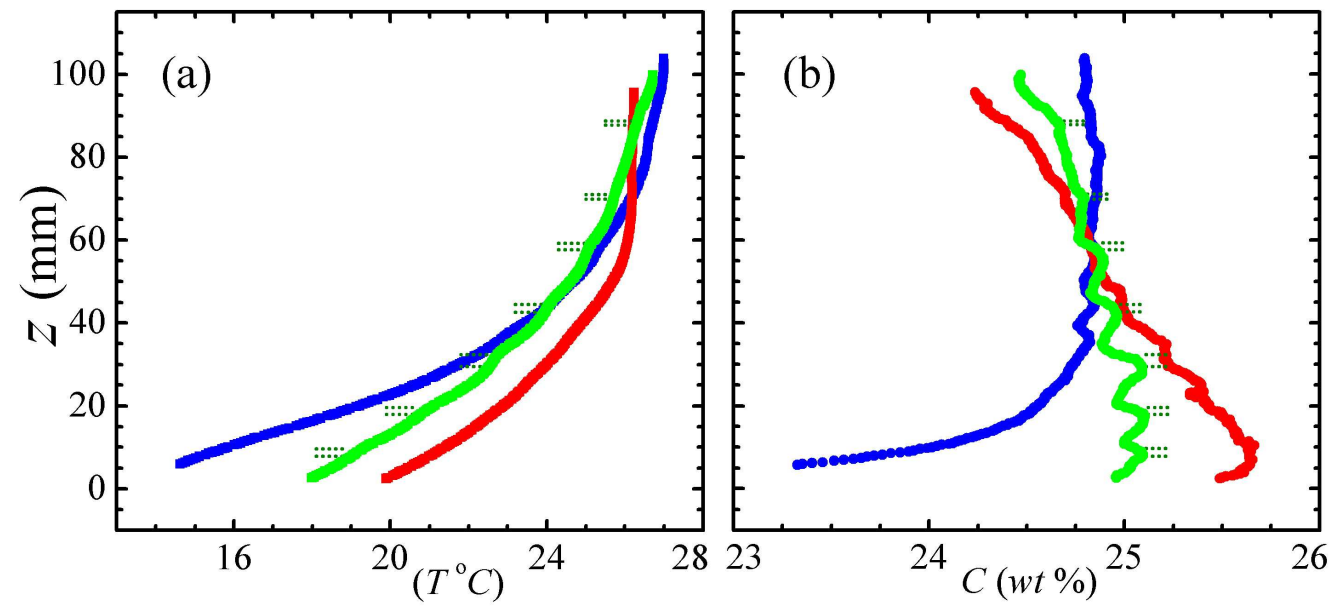

Figure 11. Vertical temperature (a) and concentration (b) profiles measured in the liquid region in the middle of the 6 th modulation period with $A=10 K$ and $\tau=4000$ s. Blue curve: $C_{0}=26 w t \%$, green curve: $C_{0}=29 w t \%$, red curve: $C_{0}=30 w t \%$. Positions of the inflection points (where the vertical gradient is maximum) along the curves $C(z)$ in (b) coincide with the top and bottom interfaces of the double-diffusive layers indicated by pairs of horizontal dashed line for $C_{0}=29 w t \%$.

at late times where the dendritic structure of the mushy layer was nearly independent of time. However, measurements of Shirtcliffe et al. (1991) and Worster (1992) were performed in an early period when the growth and development of the dendritic structure in the mushy layer is significant. The accumulated effects of solute diffusion grow over time, and hence it is possible that the observed basal ramp feature may be a result of solute transport. Because of the coupling of temperature and concentration by the liquidus relation, the thermal gradient at the cold lower boundary leads to a corresponding solute gradient. Thus solutal diffusion transports solute towards the boundary. Unlike heat, solute cannot be removed through the lower boundary, and hence builds up over time, with a tendency towards local supersaturation of $\mathrm{NH}_{4} \mathrm{Cl}$ that is relieved by enhanced solidification in a diffusive boundary layer near to the base. Using a solutal diffusivity $D_{s}=1.3 \times 10^{-3} \mathrm{~mm}^{2} \mathrm{~s}^{-1}$ (Neufeld \& Wettlaufer 2008) the diffusion length after $24000 \mathrm{~s}$ is $\sqrt{D_{s} t} \approx 6 \mathrm{~mm}$. This is commensurate with the basal boundarylayer thickness in figure 10 after 6 modulation periods, lending further support to the hypothesised role of solute diffusion.

\subsection{The mean solid fractions measured in the mush and liquid region}

We also performed measurements of the concentration and temperature distributions in the liquid region during the experiment. Profiles of $C(z)$ and $T(z)$ were collected by a conductivity-temperature probe traversing vertically across the liquid region (see $\S 2$ and Fig. 1a). Figure 11a shows three sets of temperature profiles $T(z)$ for initial fluid concentrations $C_{0}=26 w t \%, 29 w t \%$ and $30 w t \%$, respectively. There is a sharp statically stable temperature gradient in the near field above the mushy layer and overall an increasing mean temperature as the height increases. With a larger $C_{0}$ we observed a smaller temperature gradient just above the mushy layer due to the higher interfacial temperature. Moreover, the temperature field becomes more uniform in the far field near the top boundary of the liquid region. Further examinations of these temperature profiles reveal several sharp changes of the temperature gradient with $z$, for large $C_{0}$. 


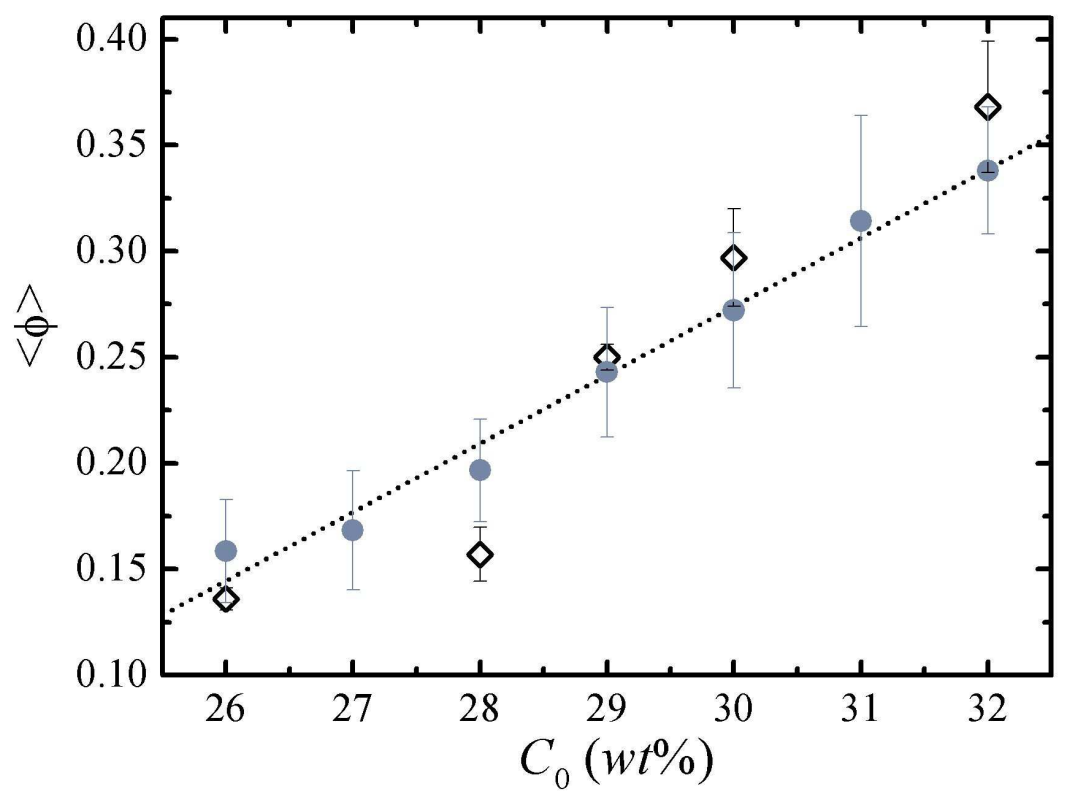

FIGURE 12. The depth-mean solid fraction in the mushy layer plotted as a function of the initial liquid concentration $C_{0}$. Results $\bar{\phi}$ are obtained from temperature data analysis (solid circles) and $\langle\phi\rangle$ from direct concentration measurement in the liquid region (open diamonds). The error bars indicate the standard deviation of $\phi_{p}-\phi$ for temperature data analysis $\bar{\phi}$, and maximum difference between measurements for the concentration method $\langle\phi\rangle$. The dotted straight line shows a linear regression $\bar{\phi}=0.0323 C_{0}[w t \%]-0.7$.

Such fine structures are superimposed on the background of a large temperature gradient in the near field, most clearly seen with $C_{0}=29 w t \%$. We ascribe the formation of these structures to double-diffusive convection that enhances local thermal mixing of the fluid within each convection cell (Fig. 1a), as discussed below.

Driven by the mushy-layer mode convection, relatively low-density fluid was continuously transported by plumes from chimneys in the mushy region, upwards through the overlying liquid region (Fig. 1a). By the late modulation periods, a stable compositional stratification is established in the liquid region, and we observed the formation of doublediffusive layers with a horizontal temperature gradient created by the cold chimney plumes. (The formation of the double-diffusive layers is through flow intrusions in the stratified background fluid driven by the horizontal thermal gradient; see discussions in Thorpe et al. 1969; Jeevaraj \& Imberger 1991; Wettlaufer et al. 1997). The signature of these double-diffusive layers is more pronounced in the concentration profiles $C(z)$ shown in Fig. 11b. We find that the vertical positions of $C(z)$ with the sharpest gradient coincide with those of the double-diffusive layer interfaces indicated by pairs of horizontal dashed line (See the Supplementary Materials in Part 1 for videos that capture the evolution of the double-diffusive layers in a state with background convection of the liquid region above a growing mushy layer, both in constant cooling and periodic cooling modes).

The results of $T(z)$ and $C(z)$ shown in Fig. 11 enable us to independently determine the mean solid fraction in the mushy layer. Assuming local thermodynamic equilibrium is reached in the mushy layer with $T=T_{L}(C)=T_{E}+\Gamma\left(C-C_{E}\right)$, then applying solute conservation to the entire depth of the tank yields 


$$
h\langle\phi\rangle+(1-\langle\phi\rangle) \int_{0}^{h}\left(\frac{T-T_{E}}{\Gamma}+C_{E}\right) d z+\int_{h}^{H} C(z) d z=C_{0} H
$$

where $H$ is the height of the tank, the solid concentration $C_{s}=1$, and $\langle\phi\rangle$ is the average solid fraction weighted by the total concentration over the mushy layer. Formally, $\langle\phi\rangle$ is defined by $C_{s}\langle\phi\rangle+\bar{C}(1-\langle\phi\rangle)=\overline{C_{s} \phi+C(1-\phi)}$ where overbars denote a vertical average over the mushy region. For each initial concentration $C_{0}$ we have made measurements of $C(z)$ by scanning the probe over the entire depth of the liquid region several times during the 6th modulation period, Results of the averaged $C(z)$ are used to determine $\langle\phi\rangle$ through (4.5).

Meanwhile we determine the mean depth-averaged solid fraction $\bar{\phi}$ by depth-integrating profiles of $\phi(z)$ that were obtained from the thermal response of the mushy layer (Fig. $9)$. Results for $\langle\phi\rangle$ and $\bar{\phi}$ obtained in both methods are shown as a functions of the initial concentration $C_{0}$ in Fig. 12. We find that for low $C_{0}$ the mean solid fraction $\bar{\phi}$ is slightly overestimated in the temperature-measurement approach, possibly because a relatively lower resolution for $\phi(z)$ was obtained in thinner mushy layers. With high initial concentration $C_{0}$, there is better agreement of $\bar{\phi}$ and $\langle\phi\rangle$. Overall, the good agreement of the two sets of data obtained from independent experimental approaches indicates that our method of temperature-data analysis is adequate to provide relatively accurate results for the mean solid fraction distribution in a mushy layer.

\section{Summary and Discussion}

This paper presents experimental studies of the distributions of temperature and solid fraction of growing mushy layers that are subjected to periodical cooling from below. A new method is presented for determining vertical profiles of solid fraction in the mushy layer based on high-resolution temperature measurements. Here we summarize the main results and discuss questions that are still open for future investigation.

The evolution of the local temperature $T(z, t)$ at various heights demonstrates that the temperature oscillations of the bottom boundary $T_{b}$ propagate through the mushy layer with substantial decay in the amplitude and phase delays. We find that the phase lag of $T(z, t)$ behind $T_{b}$ increases approximately linearly with increasing heights, which indicates a constant propagation speed of the thermal oscillations. The oscillation amplitude of $T(z, t)$ decays exponentially in depth. Both features are consistent with the classical thermal wave behaviour for diffusive transport subject to a varying surface temperature. The decay rate decreases with an increasing initial concentration, but the propagation speed increases with increasing concentration. Comparing to the thermal wave problem, these observations suggest that a higher fluid concentration leads to a greater solid fraction in average, which enhances the thermal conductivity and reduces the specific heat in the mushy layer.

We compared the scaled vertical temperature profiles $T^{*}\left(z^{*}\right)$ at different oscillation phases. The results show that a negative temperature gradient may appear near to the bottom boundary of the mush when the boundary temperature $T_{b}$ is close to minimum. We attribute this phenomenon to the finite propagation speed of the temperature oscillations through the mushy layer. This effect is more pronounced in late growth periods and with large initial concentrations, when a larger mush thickness reduces the average temperature gradient across the mushy layer. Experimental results during the late growth periods show that the temperature evolution within a modulation period is highly repeatable at each height near the bottom boundary (Fig. 2), indicating that 
the thermal properties and therefore the porosity structure of the mushy layer becomes nearly independent of time near $z=0$. The conclusion is supported as well by direct solid-fraction measurements as shown in Fig. 10. However, near the mush-liquid interface we find $\phi(z)$ is still slightly drifting in time in this stage (Fig. 10). The small variations of $\phi(z)$ near $z=h$ may be due to the brine flux carried by the chimney plumes that still exist in late modulation periods as shown in Fig. 1a, acting in concert with a slight retreat of the position of the mush-liquid interface as seen in Fig. 2d.

Through measurements of temperature we determine the various components of heat transport through the mushy layer and reveal that neither the advection heat flux nor the latent heat transfer plays an important role in the heat equation at late times, when the mushy layer is oscillating about a nearly steady background state. The heat transport through conduction is the main component that determines the rate of change of the specific heat. Based on these experimental findings, we developed a mathematical scheme to solve for the depth-dependence of the thermal conductivity and the specific heat coefficient, and hence determine the solid fraction profiles in the mushy layer for various initial concentrations. The method uses the response to small-to-moderate amplitude modulation of the boundary temperature in order to infer the material properties. In this theoretical scheme we separately consider the amplitude and the phase of terms in the heat equation, and determine the thermal diffusivity $\alpha_{m}$ from the amplitude and the associated variable $p_{m}=\left(\partial k_{m} / \partial z\right) /(c p)_{m}$ from the phase. Estimates of the solid fraction determined by these two approaches are in good agreement with each other. We note that this approach to determining $\phi$ relies on well resolved measurements of periodic temperature oscillations in the mushy layer. Because the amplitude of the thermal waves decays with height, this means that the signal-to-noise ratio will likely decrease with height through the mush. Hence solid fraction estimates very close to the mush-liquid interface may be less reliable if the thermal wave amplitude becomes comparable to convective fluctuations in the overlying fluid. The decay lengthscale $\sqrt{2 \alpha_{m} / \omega}$ scales with the diffusion length over a modulation period, and hence modulation periods approaching the diffusion time $h^{2} / \alpha_{m}$ across the full mushy layer depth will give a larger signal for probing the structure near the mush-liquid interface. The analysis also relies on a relatively small heat transport via convection within the mushy layer, and a comparatively small change in $\phi$ over a modulation period due to macroscopic solute transport (such as via gravity drainage). Understanding the impact of modulated thermal forcing on the dynamics of gravity drainage remains an open question.

Our experimental results show that profiles of the solid fraction $\phi(z)$ exhibit a clifframp-cliff structure with a rapid decrease in $\phi$ with increasing $z$ near the bottom and mush-liquid interface, but less variation in the interior of the mushy-layer. This structure is more pronounced for higher initial concentration $C_{0}$. For very low concentrations the solid fraction appears to be linearly dependent on height. The volume-average of the solid faction, and the local fluctuations in $\phi(z)$ both increase as $C_{0}$ increases. We suggest that the basal ramp-structure of the solid fraction profiles is possibly due to the diffusive transport of solute towards the bottom boundary. As a result of local thermodynamic equilibrium, the temperature gradient through the mush leads to an accompanying gradient in the liquid concentration which allows a diffusive solute flux which cannot be lost through the impermeable lower boundary. The increasing build up of solute leads to local solidification and the increasing solid fraction near the bottom boundary.

Finally we performed measurements of the fluid concentration in the liquid region. Through the conservation law for salinity, these measurements provide an independent evaluation of the mean solid fraction of the mushy layer. The agreement between both sets of experimental results suggest the adequateness of the present temperature-measurement 
approach in probing variation of the thermal properties in mushy layers. The measurements of fluid concentration and temperature also reveal interesting structures, which are broadly consistent with double-diffusive features formed by the interaction of a stable solute stratification with lateral cooling by the cold solute depleted plumes rising from the mushy layer. Whilst peripheral to this study, these features may present an interesting topic for future work.

The solid fraction $\phi$, or porosity $1-\phi$, of solidifying alloys can have an important control on the material properties of the mushy layer, including the phase-weighted thermal, electrical and mechanical properties, along with the permeability to fluid transport. The solid fraction $\phi(z)$ and liquid concentration $C(z)$ also control the bulk phase weighted composition of alloys that are rapidly quenched in industrial solidification processes (Copley et al. 1970). Hence, quantification of $\phi$ is relevant to a range of engineering, geophysical and geological settings (as discussed by Zhong et al. 2012). The analysis presented in $\S 4$ provides a potential methodology to infer the solid fraction $\phi$ in settings where the mushylayer is subject to small amplitude modulations in thermal forcing, and temperature measurements are readily available. An interesting potential application is the growth and evolution of sea ice, which is a mushy layer of salty brine and ice (Feltham et al. 2006). In addition to the varying material properties, the evolving porosity controls biological habitats and chemical transport within the sea-ice pore space (Hunke et al. 2011). Furthermore, estimates of $\phi(z, t)$ and $T(z, t)$ can be combined with the assumption of local thermodynamic equilibrium to estimate bulk salinity and infer a record of past salt fluxes from sea ice into the ocean (Notz \& Worster 2008) which drive mixing in the polar oceans. Temperature measurements are frequently made via thermistor strings embedded within boreholes in the evolving ice (c.f. Richter-Menge et al. 2006; Jackson et al. 2013) but non-destructive estimates of solid fraction are more challenging and less common. Sea ice is subject to fluctuating atmospheric and oceanic forcing on a variety of timescales (including a diurnal cycle in spring and summer, which is shorter than the seasonal evolution of sea ice thickness). This may offer a potential pathway to infer the solid fraction of sea ice from the response to fluctuating thermal forcing (although further work is needed to disentangle the response to the broad range of frequencies usually experienced in environmental settings). We hope that the results here provide useful insight for developing measurements of the solid fraction of mushy layers, both in sea ice and other environmental and engineering settings.

\section{Acknowledgement}

This work was supported by the National Science Foundation of China through Grant No. 11572230 and 11772235, and a NSFC/RGC Joint Grant No. 11561161004. J.-Q. Z. acknowledges supports from the Fundamental Research Funds for the Central Universities (Grant No. 22120180115 and 22120180280). G.-Y. D. was supported partially by a NSFC/RGC Joint Grant No. CUHK437/15. A.J.W. acknowledges European Union Award PCIG13-GA-2013-618610 SEA-ICE-CFD, and thanks the Isaac Newton Institute for Mathematical Sciences for hospitality during the programme Mathematics of Sea Ice Phenomena (EPSRC Grant EP/K032208/1). 


\section{Appendix A. Neglect of latent heating}

In this appendix, we estimate the likely magnitude of the error incurred by neglecting the latent heating term in the analysis of section 4. From (4.1), the heat equation yields

$$
c p_{m} \frac{\partial T}{\partial t}=\frac{\partial}{\partial z}\left(k_{m} \frac{\partial T}{\partial z}\right)+L \frac{\partial \phi}{\partial t}
$$

where we have neglected convective heat transport $(w=0)$ following the analysis in $\S 4$. Figure 10 suggests that $\phi$ varies little between modulation periods, so that $\phi(t)$ has no significiant long term trend (but could still vary periodically in time over a modulation period). We now estimate the contribution of periodic modulation of $\phi$. In part I (Ding et al. 2018) we estimated $\partial \phi / \partial t$ from solute conservation, neglecting solute transport at early times before there is significant modification of the bulk concentration by gravity drainage. Motivated by the lack of trend in $\phi(t)$ in figure 10 between modulation periods, we again assume that solute transport is weak at late times, so that the bulk phaseweighted solute concentration

$$
C_{\text {bulk }}(z)=\phi C_{s}+(1-\phi) C(z, t),
$$

is locally constant in time, at late times. In constructing this argument we allow for the possibility that the bulk concentration may vary with height with $C_{\text {bulk }}(z)<C_{0}$, as a result of solute fluxes from gravity drainage at earlier times. The condition (A 2) rearranges to yield

$$
\phi=\frac{C_{b u l k}-C_{L}(T)}{C_{S}-C_{L}(T)}=\frac{T_{L}\left(C_{b u l k}\right)-T}{T_{L}\left(C_{S}\right)-T},
$$

where $T=T_{L}(C)=T_{E}+\Gamma\left(C-C_{E}\right)$ is the assumed linear liquidus relation in the mushy layer, with inverse $C=C_{L}(T)$. Taking the derivative of (A 3) and using the chain rule yields

$$
\frac{\partial \phi}{\partial t}=\frac{d \phi}{d T} \frac{\partial T}{\partial t}, \quad \text { with } \quad \frac{d \phi}{d T}=\frac{\phi-1}{T_{L}\left(C_{s}\right)-T} .
$$

Using (A 4) to eliminate $\partial \phi / \partial t$ from (A 1) results in a nonlinear diffusion equation for $T$

$$
c p_{\text {eff }} \frac{\partial T}{\partial t}=\frac{\partial}{\partial z}\left(k_{m} \frac{\partial T}{\partial z}\right), \quad \text { where } c p_{\text {eff }}=c p_{s} \phi+c p_{l}(1-\phi)+\frac{L(1-\phi)}{T_{L}\left(C_{s}\right)-T}
$$

is an effective heat capacity. Thus the latent heating term provides an equivalent effect to modifying the liquid heat capacity by an additive factor $L /\left[T_{L}\left(C_{s}\right)-T\right]$. Using $L=0.428 \mathrm{~J} \mathrm{~mm}^{-3}, \Gamma=4.72{ }^{\circ} \mathrm{C} / \mathrm{wt} \%, T_{E}=-15.4{ }^{\circ} \mathrm{C}, C_{E}=19.7 \mathrm{wt} \%$, and $C_{S}=100 \mathrm{wt} \%$ (Neufeld \& Wettlaufer 2008), then an approximate temperature range of $-10^{\circ} \mathrm{C}<T<20^{\circ} \mathrm{C}$ within the mushy layer (see figure 2) results in $1.15 \times 10^{-3} \mathrm{~J} \mathrm{~mm}^{-3} \mathrm{~K}^{-1}<L /\left[T_{L}\left(C_{s}\right)-T\right]<1.25 \times 10^{-3} \mathrm{~J} \mathrm{~mm}^{-3} \mathrm{~K}^{-1}$. This corresponds to $30-34 \%$ of the liquid specific heat $c p_{l}$ from table 1 , so that setting $\partial \phi / \partial t=0$ has a similar effect to an order $30 \%$ change in the liquid heat capacity. Propagating this error through the estimate of $\phi(z)$ from $\alpha_{m}$ yields a potential underestimate of $\phi$ no larger than $\Delta \phi=0.1$, which is comparable to the error bars in Fig. 9.

\section{Appendix B. Thermal wave solution with constant material properties}

In this appendix, we outline the derivation of a diffusive thermal wave behaviour arising from modulation of the boundary temperature. To yield an analytically tractable 
problem that captures the essence of the full system, we approximate $c p_{\text {eff }}$ and $k_{m}$ as constants in (A 5), and define a constant diffusivity $\alpha=k_{m} / c p_{\text {eff }}$. In contrast to the early time behaviour considered in part I (Ding et al. 2018), we consider a late time limit where the mushy layer oscillates about a steady state of thickness $h_{0}$ where conduction of heat through the mushy layer balances the heat flux $F_{T}$ from the overlying liquid. We further consider a limit with high frequency modulation $\omega h_{0}^{2} / \alpha \gg 1$, so that the modulation period $\tau=2 \pi / \omega$ is much shorter than the diffusion timescale $h_{0}^{2} / \alpha$ across the mushy layer depth. To build insight into the impact of boundary modulation we use a perturbative approach, assuming the scaled modulation amplitude $\epsilon=A / \Delta T_{0} \ll$ 1 where $\Delta T_{0}=T_{L}\left(C_{i}\right)-T_{b, 0}$ is the temperature difference between the mush-liquid interface (with concentration $C_{i}$ ) and the mean basal temperature $T_{b, 0}$. One can replace $T_{L}\left(C_{i}\right) \approx T_{L}\left(C_{0}\right)$ below with reasonable accuracy. The separation of scales is marginal since $0.18 \leqslant \epsilon \leqslant 0.53$ for $5^{\circ} \mathrm{C} \leqslant A \leqslant 15^{\circ} \mathrm{C}$ and $\Delta T_{0}=28.5^{\circ} \mathrm{C}$, but we still find that the expansion provides useful insight.

With the assumed constant material properties, (A 5) yields

$$
\frac{\partial T}{\partial t}=\alpha \frac{\partial^{2} T}{\partial z^{2}}
$$

which is subject to boundary conditions

$$
T=T_{b, 0}+\epsilon \Delta T_{0} \sin \left(\omega t+\phi_{0}\right) \quad \text { at } \quad z=0, \quad T=T_{b, 0}+\Delta T_{0} \quad \text { at } \quad z=h .
$$

and the Stefan condition

$$
\mathcal{S} \Delta T_{0} \frac{d h}{d t}=\left.\alpha \frac{\partial T}{\partial z}\right|_{z=h}-\frac{F_{T}}{c p_{m}}, \quad \mathcal{S}=\frac{c p_{l}\left[T_{l}-T_{L}\left(C_{l}\right)\right]+L \phi_{i}}{c p_{\text {eff }} \Delta T_{0}},
$$

where $T_{l}$ is the far field temperature of the overlying liquid, and $\phi_{i}$ the assumed interfacial solid fraction (c.f. equations 3.4, 3.6 and 3.7 in Part I, Ding et al. 2018). Expanding $T=T_{0}+\epsilon T_{1}+\ldots$ and $h=h_{0}+\epsilon h_{1}+\ldots$ we find that the leading-order problem at $O\left(\epsilon^{0}\right)$ is

$$
\frac{\partial T_{0}}{\partial t}=\alpha \frac{\partial^{2} T_{0}}{\partial z^{2}}, \quad T_{0}=T_{b, 0} \quad \text { at } \quad z=0, \quad T_{0}=T_{b, 0}+\Delta T_{0} \quad \text { at } \quad z=h_{0} .
$$

The long-time steady-state solution to (B 4) and (B 3) is

$$
T_{0}=T_{b, 0}+\Delta T_{0} \frac{z}{h_{0}}, \quad h_{0}=\frac{\alpha c p_{\mathrm{eff}} \Delta T_{0}}{F_{T}},
$$

where the steady mush thickness $h_{0}$ is maintained by the balance between conduction and the heat flux $F_{T}$ in the liquid.

At $O(\epsilon)$, the linearised perturbations to (B 1) and (B 2) satisfy

$$
\frac{\partial T_{1}}{\partial t}=\alpha \frac{\partial^{2} T_{1}}{\partial z^{2}}, \quad T_{1}=\Delta T_{0} \sin \left(\omega t+\phi_{0}\right) \quad \text { at } \quad z=0, \quad T_{1}+h_{1} \frac{\partial T_{0}}{\partial z}=0 \quad \text { at } \quad z=h_{0},
$$

with the linearised perturbation to (B 3) yielding

$$
\Delta T_{0} \mathcal{S} \frac{d h_{1}}{d t}=\left.\alpha \frac{\partial T_{1}}{\partial z}\right|_{z=h_{0}}+\left.h_{1} \alpha \frac{\partial^{2} T_{0}}{\partial z^{2}}\right|_{z=h_{0}} .
$$

Note that we have here Taylor expanded functions of the form $f\left(h_{0}+\epsilon h_{1}\right)=f\left(h_{0}\right)+$ $\epsilon h_{1} f^{\prime}\left(h_{0}\right)+\ldots$ for $\epsilon \ll 1$. We analyse the system (B 6)-(B 7) in the limit of high frequency modulation $\omega h_{0}^{2} / \alpha \gg 1$. We solve using complex variable methods, setting 
$T_{1}=\operatorname{Im}\left[\tilde{T}(z) \mathrm{e}^{i \omega t+i \phi_{0}}\right]$ and $h_{1}=\operatorname{Im}\left[\tilde{h} \mathrm{e}^{i \omega t+i \phi_{0}}\right]$ for complex $\tilde{T}$ and $\tilde{h}$. Substituting this ansatz into (B 7) and eliminating derivatives of $T_{0}$ using (B 5) one can show that

$$
\tilde{h}=\left.\frac{\alpha}{i \omega \mathcal{S}} \frac{1}{\Delta T_{0}} \frac{\partial \tilde{T}}{\partial z}\right|_{z=h_{0}}
$$

Hence (B 6) reduces to

$$
\frac{i \omega}{\alpha} \tilde{T}=\frac{\partial^{2} \tilde{T}}{\partial z^{2}}, \quad \frac{\tilde{T}}{\Delta T_{0}}=1 \quad \text { at } \quad z=0, \quad \frac{\tilde{T}}{\Delta T_{0}}=0+O\left(\frac{1}{\mathcal{S}} \frac{\alpha}{\omega h_{0}^{2}}\right) \quad \text { at } \quad z=h_{0},
$$

where we again use (B 5) to eliminate $T_{0}$.

Neglecting the terms of $O\left(\alpha / \omega h_{0}^{2}\right)$, the solution to (B 9) is

$$
\frac{\tilde{T}}{\Delta T_{0}}=\frac{\mathrm{e}^{-m z}-\mathrm{e}^{-m\left(2 h_{0}-z\right)}}{1-\mathrm{e}^{-2 m h_{0}}}, \quad m=(1+i) \sqrt{\frac{\omega}{2 \alpha}} .
$$

This is easiest to interpret by exploiting the limit $\left|m h_{0}\right|=\sqrt{\omega h_{0}^{2} / \alpha} \gg 1$, from which we recover the classical thermal wave solution for diffusion in a deep layer with sinusoidally varying boundary temperature (e.g. see section 2.6 of Carslaw \& Jaeger 1959) with $\tilde{T}=\Delta T_{0} \mathrm{e}^{-m z}$ and

$$
T=T_{0}(z, t)+A \mathrm{e}^{-z \sqrt{\omega / 2 \alpha}} \sin \left(\omega t-z \sqrt{\frac{\omega}{2 \alpha}}+\phi_{0}\right) .
$$

The amplitude of the perturbation decays exponentially with e-folding length scale $\sqrt{2 \alpha / \omega}$ set by the approximate lengthscale for diffusion over a modulation cycle, whilst the phase $z \sqrt{\omega / 2 \alpha}$ varies linearly with height over the same lengthscale, which recovers the internal thermal variation seen in figure 4 .

\section{REFERENCES}

Aussillous, P., Sederman, A. J., Gladden, L. F. \& Huppert, H. E. amd Worster, M. G. 2006 Magnetic resonance imaging of structure and convection in solidifying mushy layers. J. Fluid Mech. 552, 99-125.

Backstrom, L. G. E. \& Eicken, H. 2006 Capacitance probe measurements of brine volume and bulk salinity in first-year sea ice. Cold Regions Science and Technology 46 (3), 167180.

Beckermann, C. \& Viskanta, R. 1988 Double-diffusive convection due to melting. Int. J. Hear Mass Transfer. 31, 2077-2089,.

Carslaw, H. S. \& Jaeger, J. C. 1959 Conduction of heat in solids, 2nd edn. Oxford University Press.

Chalmers, B. 1964 Principles of solidification. Wiley.

Chen, C. F. 1995 Experimental study of convection in a mushy layer during directional solidification. J. Fluid Mech. 293 (-1), 81.

Chen, C. F. \& Chen, F. 1991 Experimental study of directional solidification of aqueous ammonium chloride solution. J. Fluid Mech. 227 (-1), 567.

Chen, FALin 1997 Formation of double-diffusive layers in the directional solidification of binary solution. J. Crystal Growth 179, 277-286.

Chiareli, A.O.P. \& Worster, M.G. 1992 On measurement and prediction of the solid fraction within mushy layers. J. Cryst. Growth 125 (3-4), 487-494.

Copley, S., Giamei, A, Johnson, S. \& Hornbecker, M. 1970 The origin of freckles in unidirectionally solidified castings. Metallurgical and Materials Transactions B 1 (12), 3455-3455.

Ding, G. Y., Wells, A. J. \& Zhong, J.-Q. 2018 Solidification of binary aquaous solutions 
under periodic cooling: Part 1: Dynamics of mushy-layer growth. submitted to J. Fluid Mech. .

Eicken, H, Bock, C, Wittig, R, Miller, H \& Poertner, H. O 2000 Magnetic resonance imaging of sea-ice pore fluids: methods and thermal evolution of pore microstructure. Cold Regions Science and Technology 31 (3), 207-225.

Feltham, D. L., Untersteiner, N., Wettlaufer, J. S. \& Worster, M. G. 2006 Sea ice is a mushy layer. Geophys. Res. Lett. 33.

Golden, K. M., Eicken, H., Heaton, A. L., Miner, J., Pringle, D. J. \& Zhu, J. 2007 Thermal evolution of permeability and microstructure in sea ice. Geophysical Research Letters 34 (16).

Hallworth, Mark A. \& Huppert, Herbert E. 2004 Crystallization and layering induced by heating a reactive porous medium. Geophys. Res. Lett. 31, L13605.

Hallworth, Mark A., Huppert, Herbert E. \& Woods, Andrew W. 2005 Dissolutiondriven convection in a reactive porous medium. J. Fluid Mech. 535, 255-285.

HEAD, M. J. 1983 The use of miniature four-electrode conductivity probes for high resolution measurement of turbulent density or temperature variations in salt-stratified water flows. University of California, San Diego.

Hobbs, P. V. 2010 Ice physics. Oxford University Press.

Huguet, Ludovic, Alboussiere, Thierry, Bergman, Michael I., Deguen, Renaud, Labrosse, Stephane \& Lesceur, Germain 2016 Structure of a mushy layer under hypergravity with implications for earth's inner core. Geophys. J. Int. 204, 1729-1755.

Hunke, E. C., Notz, D., Turner, A. K. \& Vancoppenolle, M. 2011 The multiphase physics of sea ice: a review for model developers. The Cryosphere 5 (4), 989-1009.

Hunkeler, P. A., Hendricks, S., Hoppmann, M., Farquharson, C. G., Kalscheuer, T., Grab, M., Kaufmann, M. S., Rabenstein, L. \& Gerdes, R. 2015 Improved 1D inversions for sea ice thickness and conductivity from electromagnetic induction data: Inclusion of nonlinearities caused by passive bucking Multifrequency EM sea ice inversions. Geophysics 81 (1), WA45.

Huppert, H. E. 1990 The fluid mechanics of solidification. J. Fluid Mech. 212 (-1), 209.

Huppert, H. E. \& Worster, M. G. 1985 Dynamic solidification of a binary melt. Nature 314, 703-707.

Jackson, K., Wilkinson, J., Maksym, T., Meldrum, D., Beckers, J., HaAs, C. \& MACKenzie, D. 2013 A novel and low-cost sea ice mass balance buoy. Journal of Atmospheric and Oceanic Technology 30 (11), 2676-2688.

Jeevaraj, C. G. \& Imberger, J. 1991 Experimental study of double-diffusive instability in sidewall heating. J. Fluid Mech. 222, 565-586.

Lieb-Lappen, R. M., Golden, E. J. \& Obbard, R. W. 2017 Metrics for interpreting the microstructure of sea ice using x-ray micro-computed tomography. Cold Regions Science and Technology 138, 24-35.

Loper, D. E. \& Roberts, P. H. 1983 Compositional convection and the gravitationally powered dynamo. the SAO/NASA Astrophysics Data System.

Neufeld, J. A. \& Wettlaufer, J. S. 2008 An experimental study of shear-enhanced convection in a mushy layer. J. Fluid Mech. 612, 363-385.

Notz, D., Wettlaufer, J. S. \& Worster, M. G. 2005 A non-destructive method for measuring the salinity and solid fraction of growing sea ice in-situ. J. Glaciol. 51, 159-166.

Notz, D. \& Worster, M. G. 2008 In situ measurements of the evolution of young sea ice. J. Geophys. Res. Ocean. 113 (3).

Notz, D. \& Worster, M. G. 2009 Desalination processes of sea ice revisited. J. Geophys. Res. Ocean. $114(5), 1-10$.

Peppin, S. S. L., Huppert, H. E. \& Worster, M. G. 2008 Steady-state solidification of aqueous ammonium chloride. J. Fluid Mech. 599, 465-476.

Pesci, A. I., Porter, M. A. \& Goldstein, R. E. 2003 Inertially driven buckling and overturning of jets in a hele-shaw cell. Phys. Rev. E. 68, 056305.

Pringle, D. J., Eicken, H., Trodahl, H. J. \& Backstrom, L. G. E. 2007 Thermal conductivity of landfast antarctic and arctic sea ice. Journal of Geophysical Research: Oceans 112 (C4). 
Rees Jones, D. \& Worster, M. 2013 Fluxes through steady chimneys in a mushy layer during binary alloy solidification. J. Fluid Mech. 714, 127.

Richter-Menge, J. A., Perovich, D. K., Elder, B. C., Claffey, K., Rigor, I. \& ORTMEYER, M. 2006 Ice mass-balance buoys: a tool for measuring and attributing changes in the thickness of the arctic sea-ice cover. Annals of Glaciology 44, 205-210.

Rosenberger, F.E. 1979 Fundamentals of Crystal Growth I: Macroscopic Equilibrium and Transport Concepts. Springer Berlin Heidelberg.

Sampson, C., Golden, K. M., Gully, A. \& Worby, A. P. 2011 Surface impedance tomography for antarctic sea ice. Deep Sea Research Part II: Topical Studies in Oceanography $\mathbf{5 8}$ (9), 1149-1157.

Shirtcliffe, T.G.L., Huppert, Herbert E. \& Worster, M.Grae 1991 Measurement of the solid fraction in the crystallization of a binary melt. J. Cryst. Growth 113 (3-4), 566-574.

TAit, S. \& JAUPART, C. 1989 Compositional convection in viscous melts. Nature 338 (6216), $571-574$.

Thorpe, S. A., Hutt, P. K. \& Soulsby, R. 1969 Ithe effect of horizontal gradients on thermohaline convection. J. Fluid Mech. 38, 375-400.

Wells, A. J., Wettlaufer, J. S. \& Orszag, S. A. 2010 Maximal potential energy transport: A variational principle for solidification problems. Physical Review Letters 105 (25), 1-4.

Wettlaufer, J. S., Worster, M. G. \& Huppert, H. E. 1997 Natural convection during solidification of an alloy from above with application to the evolution of sea ice. J. Fluid Mech. 344, 291-316.

Worster, M. G. 1986 Solidification of an alloy from a cooled boundary. J. Fluid Mech. 167, 481-501.

Worster, M. G. 1991 Natural convection in a mushy layer. J. Fluid Mech. 224 (-1), 335.

Worster, M G. 1992 On Measurement and Prediction of the Solid Fraction Within Mushy Layers. J. Cryst. Growth 125, 487-494.

Worster, M. G. 1997 Convection in mushy layers. Annual Review of Fluid Mechanics 29, 91-122.

Worster, M. G. 2000 in Perspectives in fluid dynamics: a collective introduction to current research, pp. 393-446. Cambridge: Cambridge University Press.

Yu, J., Bergman, M. I., Huguet, L. \& Alboussiere, T. 2015 Partial melting of a Pb-Sn mushy layer due to heating from above, and implications for regional melting of Earth's directionally solidified inner core. Geophys. Res. Lett. 42, 7046-7053.

Zhong, J.-Q., Fragoso, A. T., Wells, A. J. \& Wettlaufer, J. S. 2012 Finite-samplesize effects on convection in mushy layers. J. Fluid Mech. 704 (2), 89-108, arXiv: arXiv:1201.2070v1. 